\title{
DNA replication stress mediates APOBEC3 family mutagenesis in breast cancer
}

\author{
Nnennaya Kanu ${ }^{1 \dagger}$, Maria Antonietta Cerone ${ }^{1 \dagger}$, Gerald Goh¹, Lykourgos-Panagiotis Zalmas², Jirina Bartkova, \\ Michelle Dietzen ${ }^{1}$, Nicholas McGranahann ${ }^{2}$, Rebecca Rogers ${ }^{5}$, Emily K. Law ${ }^{6}$, Irina Gromova ${ }^{3}$, Maik Kschischo ${ }^{7}$, \\ Michael I. Walton ${ }^{5}$, Olivia W. Rossanese ${ }^{5}$, Jiri Bartek ${ }^{3,4}$, Reuben S. Harris ${ }^{6}$, Subramanian Venkatesan ${ }^{1,2^{*}}$ \\ and Charles Swanton ${ }^{1,2^{*}}$
}

\begin{abstract}
Background: The APOBEC3 family of cytidine deaminases mutate the cancer genome in a range of cancer types. Although many studies have documented the downstream effects of APOBEC3 activity through next-generation sequencing, less is known about their upstream regulation. In this study, we sought to identify a molecular basis for APOBEC3 expression and activation.

Results: HER2 amplification and PTEN loss promote DNA replication stress and APOBEC3B activity in vitro and correlate with APOBEC3 mutagenesis in vivo. HER2-enriched breast carcinomas display evidence of elevated levels of replication stress-associated DNA damage in vivo. Chemical and cytotoxic induction of replication stress, through aphidicolin, gemcitabine, camptothecin or hydroxyurea exposure, activates transcription of $A P O B E C 3 B$ via an ATR/Chk1-dependent pathway in vitro. APOBEC3B activation can be attenuated through repression of oncogenic signalling, small molecule inhibition of receptor tyrosine kinase signalling and alleviation of replication stress through nucleoside supplementation.

Conclusion: These data link oncogene, loss of tumour suppressor gene and drug-induced replication stress with APOBEC3B activity, providing new insights into how cytidine deaminase-induced mutagenesis might be activated in tumourigenesis and limited therapeutically.
\end{abstract}

Keywords: APOBEC, Genomic instability, Replication stress, Somatic mutation

\section{Significance}

APOBEC3 mutates the cancer genome in a broad range of cancer types. In this study we link DNA replication stress mediated by oncogene activation or cytotoxic exposure to APOBEC3B activity. These insights provide support for therapeutic approaches that might limit the activity of this mutagenic process.

\footnotetext{
*Correspondence: subramanian.venkatesan@crick.ac.uk; charles.swanton@ crick.ac.uk

${ }^{\dagger}$ Equal contributors

${ }^{1}$ UCL Cancer Institute, CRUK Lung Cancer Centre of Excellence, Paul O'Gorman Building, Huntley St., London, UK

Full list of author information is available at the end of the article
}

\section{Background}

Genomic instability is a well-recognized hallmark of cancer and is known to cause both aberrant chromosome architecture as well as mutational changes at the single nucleotide level [1]. We have previously identified a role for DNA replication stress in human tumourigenesis [2, 3] and in the generation of chromosomal instability, which contributes to intratumour heterogeneity $[4,5]$. More recently, analyses performed in over 30 cancer types have identified that many tumours exhibit cytosine mutation biases, particularly $\mathrm{C}$ to $\mathrm{T}$ transitions and $\mathrm{C}$ to $\mathrm{G}$ transversions predominantly in TCA or TCT trinucleotide contexts [6-9]. The mutagen has been identified as 
a member of the apolipoprotein B mRNA editing enzyme, catalytic polypeptide-like 3 (APOBEC3) family of cytidine deaminases [9-11]. We recently described the enrichment of APOBEC3 mutagenesis later in tumour evolution, occurring as subclonal mutations in estrogen receptor (ER)-negative breast cancer, lung adenocarcinoma, head and neck squamous carcinoma and bladder carcinoma, suggesting APOBEC3 may contribute to branched evolution in some tumour types [12-14].

Although, the involvement of APOBEC3 in cancer has been refined over the past few years, the functional regulation of this family of enzymes is yet to be fully understood. A closer examination of kataegis in cancer samples revealed that APOBEC3-induced mutations often colocalised with breakpoint rearrangements, and within breast cancer, the HER2-enriched (HER2+) subtype has been shown to display evidence of APOBEC3-mediated mutagenesis [9]. Furthermore, HER2+ breast cancer is associated with high levels of somatic copy number aberrations (SCNAs) [9]. Whether there is a mechanistic connection between the underlying causes of chromosomal copy number aberrations and the generation of APOBEC3 mutagenesis in HER2+ breast cancer has not been explored.

The extent of hypermutation is likely to be dependent on both the level of APOBEC3 protein and the availability of single-stranded DNA (ssDNA) substrate [9]. In the presence of cellular cytidine deaminase, however, the rate-limiting step is thought to be substrate availability [15]. It is thought that segmental SCNA breakpoints could potentially expose more ssDNA, which is the ideal substrate for APOBEC3 $[15,16]$. The availability of ssDNA substrate can be modulated by regulating replication fork stability and collapse [17]. Additional processes that induce ssDNA exposure include oncogene-induced replication stress, double strand break (DSB) repair [18], R-loops formed during transcription [19] and telomere crisis [20]. ssDNA can also be exposed by DNA end resection during DSB repair [15]. In addition, Gordenin and colleagues [16] previously identified that the extent of strand-coordinated mutation clusters in yeast was increased following exposure of cells to the chemical mutagen methyl-methanesulfonate.

In this study, we investigated the genomic correlates of APOBEC3 mutagenesis in breast cancer. We examined whether DNA damage signalling, triggered by ssDNA exposure by cytotoxic agents or oncogenic signalling, may contribute to APOBEC 3 activation and the mutational signature profile seen in breast cancer.

\section{Results}

HER2 amplification, PTEN and NF1 somatic mutations are associated with the APOBEC 3 signature

It has recently been shown that HER2-enriched (HER2+) breast cancers are associated with a high burden of mutations attributable to APOBEC3B [9]. We utilized breast cancer samples from The Cancer Genome Atlas (TCGA; $n=755$ ) [21], which were subclassified using the PAM50 algorithm into HER2+, basal, luminal A and luminal B subtypes [22], and assessed the fold enrichment of APOBEC3 signature mutations in each sample. Consistent with a previous report [9], the APOBEC3 mutagenesis pattern was significantly associated with the HER2+ subtype $\left(p\right.$ value $=1.086 \times 10^{-5}$, chi-square test; Fig. $\left.1 \mathrm{a}, \mathrm{b}\right)$. We also observed that HER2 amplification was significantly associated with 'APOBEC high' samples in the luminal A subtype (false discovery rate (FDR) $\mathrm{q}$-value $=0.075$, permutation test; see "Methods"), implicating HER2 as a driver of APOBEC3 mutagenesis in this subtype (Fig. 1c). Additionally, mutations in TP53, CDH1, NCOR1, PTEN and NF1, CCND1 amplification, as well as loss of TP53 and KMT2C were associated (FDR q-value $<0.1$, permutation test) with 'APOBEC high' samples in different breast cancer subtypes (Fig. 1c), which could explain the heterogeneity in APOBEC3 enrichment among samples within subtypes. Mutations in PIK3CA were also associated with the APOBEC3 signature, although it has been suggested that APOBEC3 activity itself is the main driver of these helical domain mutations [23]. We further observed that 'APOBEC high' tumours had a higher number of segmental SCNA breakpoints per sample compared with 'APOBEC low' tumours ( $p$ value $=0.000343$, Mann - Whitney $\mathrm{U}$ test; Additional file 1: Figure S1a).

We examined $A P O B E C 3 A, A P O B E C 3 B$ and $A P O B E C 3 G$ mRNA expression levels in a panel of 15 breast cancer cell lines (five luminal, five basal and five HER2+) by quantitative PCR (Fig. 2a). Most luminal cell lines (green) exhibited low levels of $A P O P E C 3 B$ mRNA expression, whereas most of the HER2+ (red) exhibited higher $A P O B E C 3 B$ mRNA levels (Fig. 2a). Basal cell lines (black) exhibited variable $A P O B E C 3 B$ mRNA levels (Fig. 2a). APOBEC3B expression was undetectable in SKBR3 cells, which are known to have a homozygous deletion of $A P O B E C 3 B$. The basal mRNA expression of $A P O B E C 3 A$ and $A P O B E C 3 G$ was almost undetectable in all cell lines tested (Fig. 2a). The observed mRNA expression levels were comparable to those identified in the Cancer Cell Line Encyclopedia (CCLE) dataset (Additional file 1: Figure S1b). We also examined the deamination activity present in these cell lysates determined using an oligonucleotide-based cytidine deamination assay [10] using two probes whose activity is dependent on APOBEC3B (Fig. 2b; Additional file 1: Figure S1c-f). There was a significant correlation between $A P O B E C 3 B$ expression and activity in these cell lines $(\mathrm{r}=0.8, p=0.0016$, Spearman rank correlation test; Additional file 1: Figure S1g).

Since HER2 signalling is known to drive a proliferative phenotype, which might provoke elevated DNA replicative stress (reviewed in [24]), we next investigated markers of DNA replicative stress in the 15 breast cancer cell lines. 

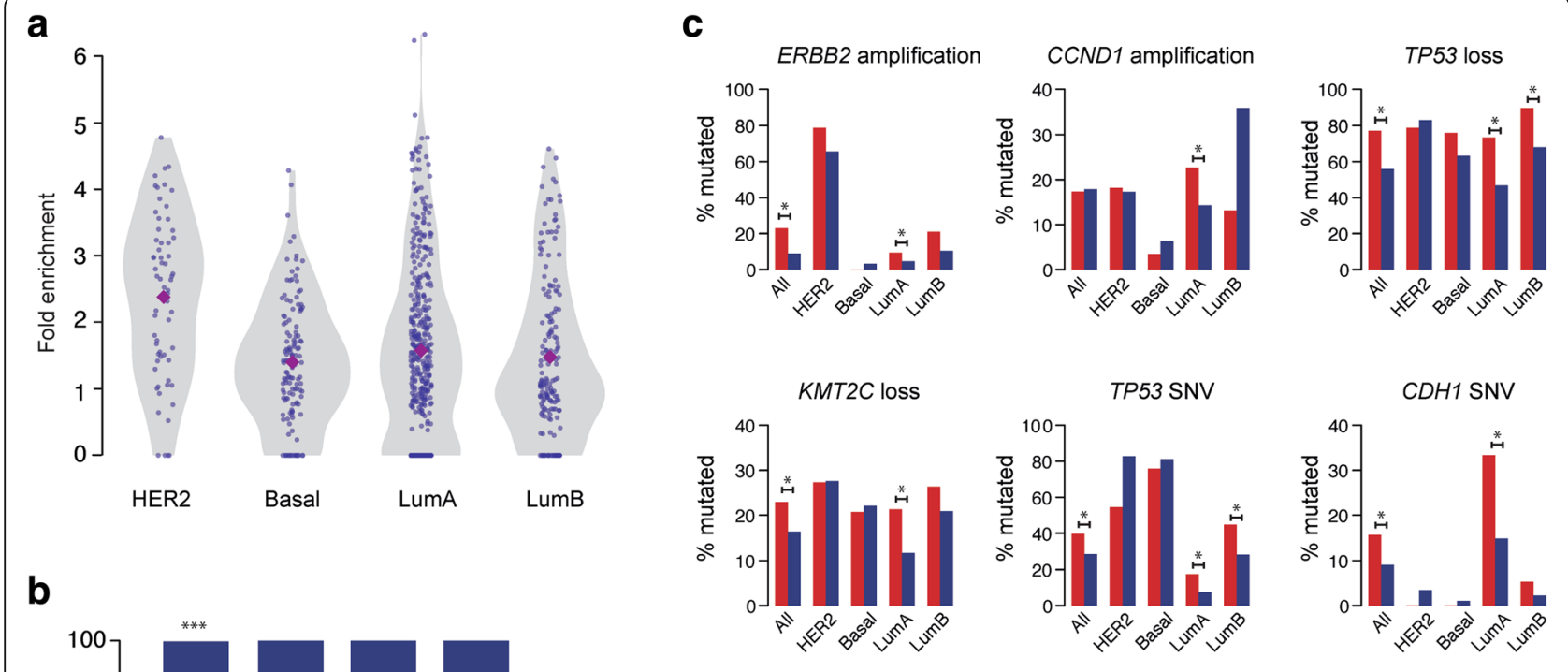

b
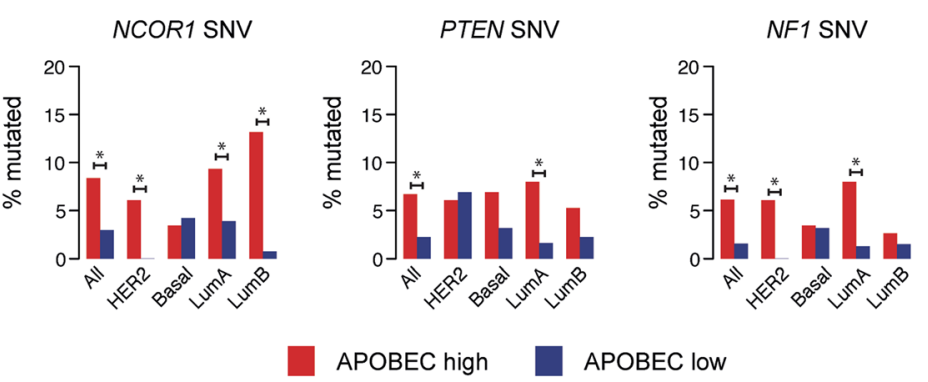

Fig. 1 APOBEC3 mutational signatures and associated genes in breast cancer subtypes. a Violin plots showing APOBEC3 mutagenesis fold enrichment. The purple diamond represents the median in each subtype. b Boxplots showing percentage of 'APOBEC high' (red) and 'APOBEC low' (blue) samples in each subtype. Asterisks represent a significant $p$ value $<0.05$ from pairwise post hoc tests. c Single-nucleotide variants (SNVs) and SCNAs associated with 'APOBEC high' tumour samples. Bars denote proportion of 'APOBEC high' (red) and 'APOBEC low' (blue) samples harbouring mutation. Amplification and loss refer to $\geq 2 \times$ ploidy and $\leq 1$ copy number relative to ploidy, respectively. An asterisk denotes significant association in subtype ( $q<0.1$ by permutation test, corrected for analysis of multiple genes by the Benjamini-Hochberg method). Note differing scales used on the $y$-axis. Lum luminal

Immunofluorescence analysis using 53BP1 nuclear bodies in G1 as a marker of DNA replication stress [25] revealed that cell lines with higher levels of $A P O B E C 3 B$ expression had significantly higher levels of replication stress $(\mathrm{r}=0.62$, $p=0.0284$, Spearman rank correlation test; Fig. 2c; Additional file 1: Figure S1h). SKBR3 (APOBEC3B null) and MDA-MB-361 (with a missense mutation in 53BP1) cell lines were both excluded from the analysis. To further confirm the relevance of DNA replication stress in APOBEC3 activation, we investigated whether alleviating replication stress would reduce APOBEC3 activity. Previously, it has been shown that supplementation of exogenous nucleosides increases the nucleotide pool in the cell and can rescue replication stress [26, 27]. Therefore, we treated BT474 cells, a HER2-amplified cell line with elevated DNA replication stress (Fig. 2c), with exogenous nucleosides prior to performing the oligonucleotide-based deamination assay. Treatment with $12.5-300 \mu \mathrm{M}$ nucleosides for $72 \mathrm{~h}$ led to a significant reduction in the basal levels of APOBEC3B protein and activity in a dose-dependent manner (Fig. 2d, e).
Supplementation of MDA-MB-134 cells (a luminal cell line with low but detectable APOBEC3 activity) with exogenous nucleosides also led to a reduction in basal APOBEC3 activity (Additional file 2: Figure S2d). No correlation was observed between reductions in cell viability in response to 300- $\mu \mathrm{M}$ nucleoside treatment (Additional file 1: Figure S1i; Additional file 3: Figure S3b) and the induction of APOBEC3 activity. Nucleoside supplementation reduced the $\mathrm{S}$ phase population in MDA-MB-134 cells but had minimal effect on the cell cycle distribution of BT474 cells (Additional file 1: Figure S1j; Additional file 4: Figure S4d). Taken together these results implicate the involvement of DNA replication stress in APOBEC3-mediated mutagenesis.

\section{Replication stress induced by cytotoxic drugs leads to APOBEC3 induction}

In order to decipher the mechanism through which replication stress is implicated in the induction of APOBEC3 activity, we tested a panel of cytotoxic drugs known to induce 


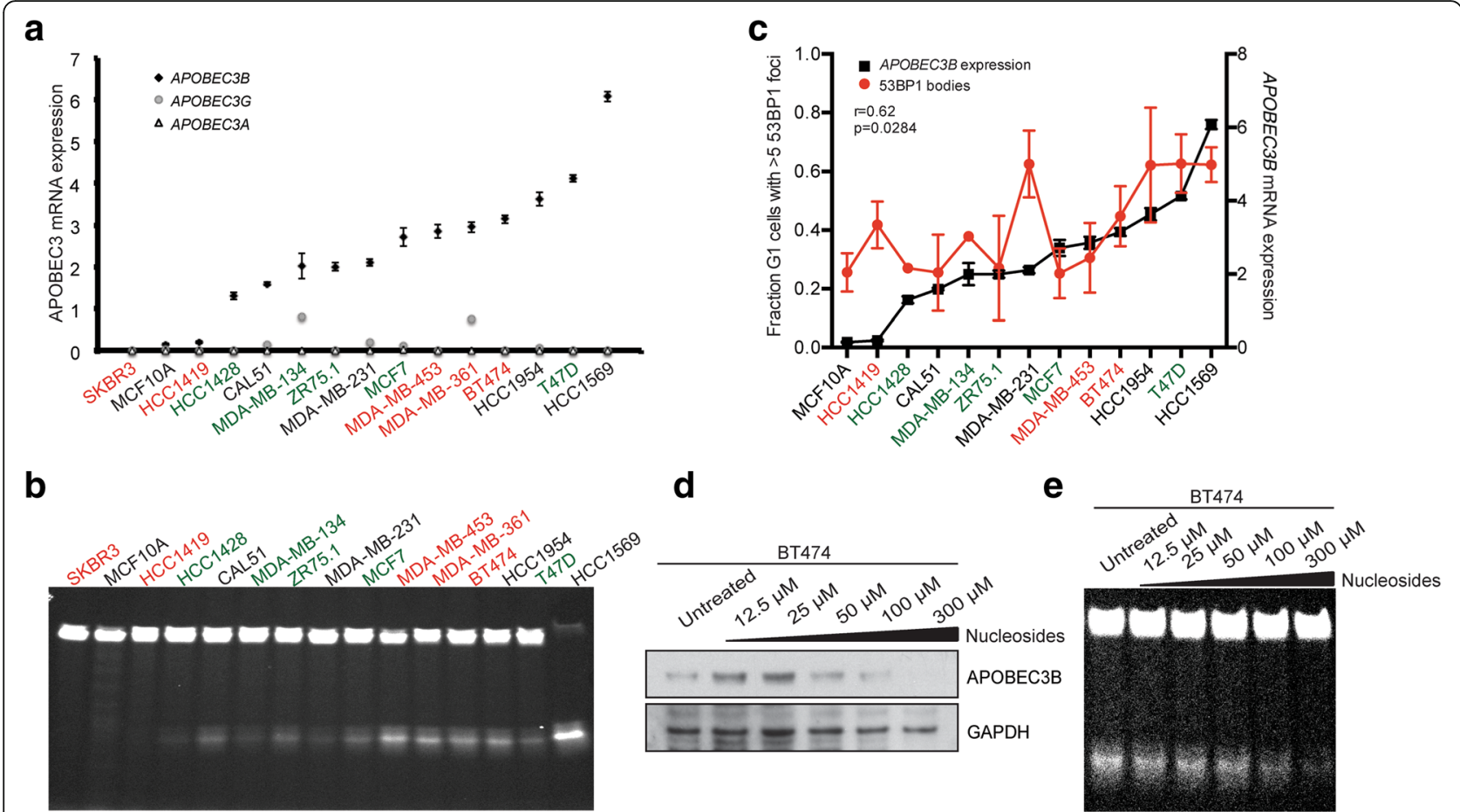

Fig. 2 APOBEC3 activity and replication stress in breast cancer cell lines. a APOBEC3B (black), APOBEC3G (grey) and APOBEC3A (white) mRNA expression in 15 breast cancer cell lines as determined by quantitative PCR. HER2+ cell lines (red), basal cell lines (black), luminal cell lines (green). SKBR3 cells have a null mutation for APOBEC3B. Error bars represent standard deviation. b APOBEC3 activity in the 15 breast cancer cell lines used in $\mathbf{a}$. Cells were lysed and subjected to oligonucleotide-based cytidine deamination assay followed by electrophoresis on $15 \%$ TBE-urea gels. c Cells were grown for two population doublings on glass coverslips followed by fixation and staining with 53BP1 and cyclin A antibodies. The fractions of cyclin A-negative cells displaying more than five 53BP1 nuclear foci were scored. APOBEC3B mRNA expression was determined by quantitative PCR from parallel cell lysates. A Spearman's rank correlation test was performed to correlate the fraction of 53BP1 nuclear bodies in cell lines with the level of $A P O B E C 3 B(r=0.62, p=$ 0.0284). Error bars represent standard deviation. $\mathbf{d}$ BT474 cells were treated with 12.5-300 $\mu \mathrm{M}$ nucleosides for $72 \mathrm{~h}$ prior to lysis. Western blots were probed with the indicated antibodies. e BT474 cells were treated as in $\mathbf{d}$ followed by lysis and an APOBEC3 cytidine deamination assay

either DSB or ssDNA damage. MCF10A cells were treated with nine drugs with broad DNA damaging or antimetabolite activity (hydroxyurea, aphidicolin, cisplatin, gemcitabine, etoposide, camptothecin, methylmethanesulfonate, doxorubicin and 5-fluorouracil) for $48 \mathrm{~h}$, after which APOBEC3 mRNA expression, protein and activity levels were assessed. Treatment of MCF10A cells with hydroxyurea, aphidicolin, gemcitabine and camptothecin elicited an increase in both $A P O B E C 3 B$ and $A P O B E C 3 G$ mRNA expression (Fig. 3a), APOBEC3B protein expression (Fig. 3b) and APOBEC3 activity (Fig. 3c; Additional file 2: Figure S2a; Additional file 5: Figure S5). Treatment of MCF7, HCC1419 and MDA-MB-134 cells with hydroxyurea, aphidicolin and gemcitabine also led to an increase in APOBEC3 activity (Additional file 2: Figure S2b-d). SKBR3 cells were included as a negative control (Additional file 2: Figure S2e). By performing the cytidine deamination assays following depletion of $A P O B E C 3 B$ by RNA interference (RNAi), we confirmed that all detectable hydroxyurea-induced deamination activity in the breast cancer cell lines was attributable to $A P O B E C 3 B$ (Additional file 2: Figure S2f, g). No correlation was observed between drug-induced cytotoxity (Additional file 3:
Figure S3a-d) and APOBEC3 activity. We observed that the four cytotoxic drugs that elicited the highest levels of APO$\mathrm{BEC} 3 \mathrm{~B}$ induction were associated with $\mathrm{S}$ phase enrichment in HCC1419 and MDA-MB-134 cells. Cell cycle arrest in MCF10A cells was also associated with an accumulation of cells at G2/M (Additional file 4: Figure S4).

In order to investigate the type of DNA damage induced by drug exposure, we assessed the extent of DSBs and ssDNA damage caused by these drugs by immunofluorescence staining of Ser139 $\gamma \mathrm{H} 2 \mathrm{AX}$ and $\mathrm{pS} 4 / 8$ replication protein A (RPA), respectively (Fig. 3d; Additional file 6: Figure S6a). There was a significant correlation between the drugs that caused the highest APOBEC BB induction and the induction of RPA phosphorylation in MCF10A cells (Additional file 6: Figure S6b-d). The four drugs that induced $A P O B E C 3 B$ activity all induced the highest levels of RPA phosphorylation in MCF10A cells (Fig. 3d), whereas in MCF7 cells this was only the case for three out of the four drugs (Additional file 6: Figure S6a). There was no correlation between drugs inducing DSBs and APOBEC3 induction. Furthermore, we observed that exposure of MCF10A cells to exogenous nucleosides also attenuated the 


\section{a}

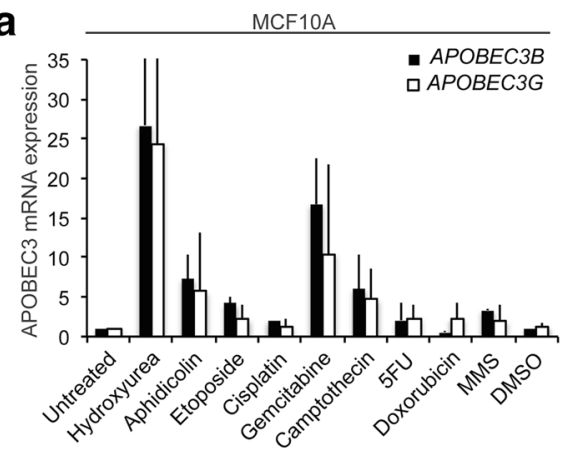

C

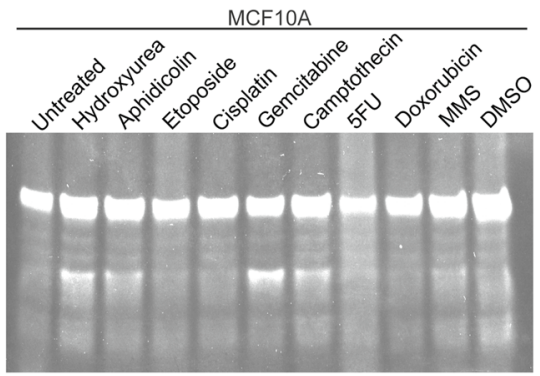

d

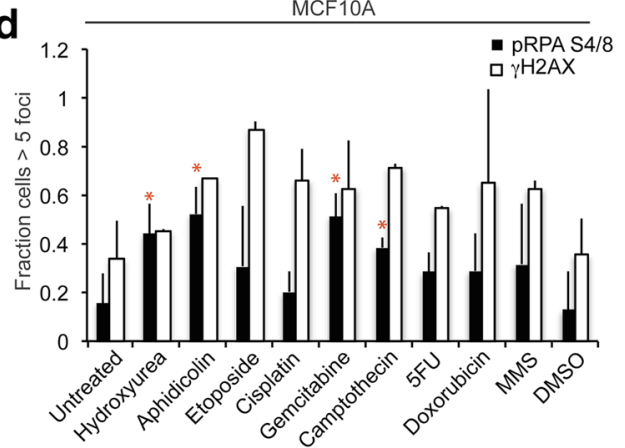

b

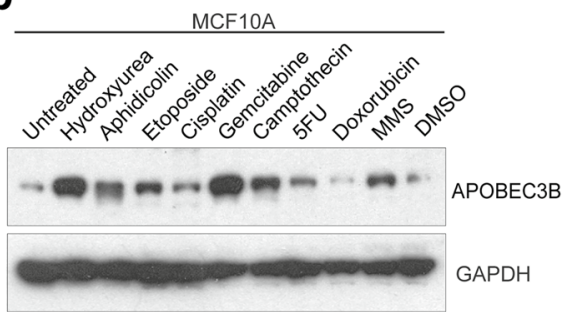

e

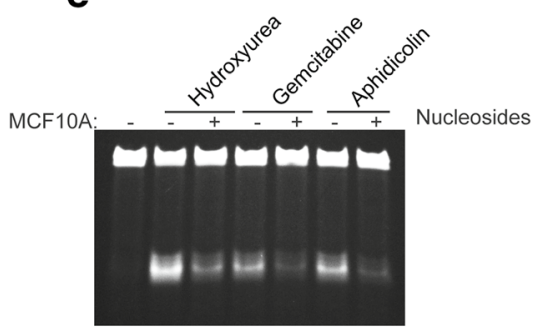

$\mathbf{f}$

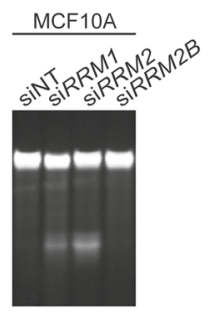

Fig. 3 Induction of replication stress and APOBEC3 activity in breast cancer cell lines. a MCF10A cells were treated with the indicated drugs for $48 \mathrm{~h}$ followed by mRNA extraction, CDNA synthesis and quantitative PCR for APOBEC3B and APOBEC3G expression levels. $\mathbf{b}$ MCF10A cells were treated as in a followed by western blotting with the indicated antibodies. C MCF10A cells were treated as in a prior to lysis and a cytidine deamination assay for APOBEC3 activity using probe 2. d MCF10A cells were treated as in a followed by fixation and immunofluorescence for Ser139 $\mathrm{YH} 2 \mathrm{AX}$ and S4/8 replication protein A phosphorylation ( $P R P A)$. Red asterisks indicate treatments inducing APOBEC $3 B$ mRNA, protein expression, activity levels and S4/8 RPA phosphorylation. e MCF10A cells were pre-treated with $300 \mu \mathrm{M}$ exogenous nucleosides followed by incubation with the indicated drugs for an additional $24 \mathrm{~h}$. Following lysis, APOBEC3 activity was measured by a cytidine deamination assay. $\mathbf{f}$ Ribonucleotide reductase subunits RRM1, RRM2 and RRM2B were depleted from MCF10A cells by RNA interference and, after $72 \mathrm{~h}$, cells were lysed and subjected to an APOBEC3 cytidine deamination assay. 5FU 5-fluorouracil, MMS methyl methanesulfonate, siNT non-targeting control siRNA

hydroxyurea, aphidicolin and gemcitabine-induced increase in APOBEC3 activity (Fig. 3e). Nucleoside supplementation reduced the hydroxyurea-induced $\mathrm{S}$ phase enrichment in MCF10A cells (Additional file 3: Figure S3e). These results suggest that DNA replication stress is able to increase APOBEC3 transcription levels and trigger its activity.

Hydroxyurea is an inhibitor of ribonucleotide reductase, an enzyme that catalyses the reduction of ribonucleotides to deoxyribonucleotides, which are required for DNA replication. Depletion of ribonucleotide reductase stalls the
DNA polymerase at the replication forks, resulting in DNA replication stress [28]. In order to further confirm the role for DNA replication stress in APOBEC3 regulation, we depleted ribonucleotide reductase subunits RRM1, RRM2 and $R R M 2 B$ by small interfering RNA (siRNA) in MCF10A cells since they exhibit low levels of basal APOBEC3B activity and replication stress. Knockdown of either RRM1 or RRM2 subunits by siRNA led to an increase in APOBEC3B deamination activity (Fig. 3f; Additional file 6: Figure S6e). Knockdown of $R R M 2 B$ encoding the small subunit of p53 
inducible ribonucleotide reductase had no effect. These results suggest a relationship between the induction of ssDNA and APOBEC3B induction.

\section{HER2 expression and PTEN knockdown contribute to APOBEC3 activity}

Having observed increased replication stress and APOBEC3 activity in many of the HER2 + cell lines, we next investigated the consequence of HER2 depletion on APOBEC3 activity using the oligonucleotide-based deamination assay.
Silencing HER2 by RNAi in HER2+ BT474 and MDA-MB361 cells led to a reduction in $A P O B E C 3 B$ mRNA expression, protein levels and deamination activity (Fig. 4a-c; Additional file 7: Figure S7a). To further examine whether this reduction was dependent on the presence of HER2 protein or on HER2 downstream signalling, $A P O B E C 3 B$ mRNA expression, protein and activity levels were assessed following exposure of HER2 + cells to HER2 tyrosine kinase inhibitors. A 24-h treatment of BT474 cells with 10 $\mathrm{nM}$ afatinib and $30 \mathrm{nM}$ lapatinib resulted in reduced

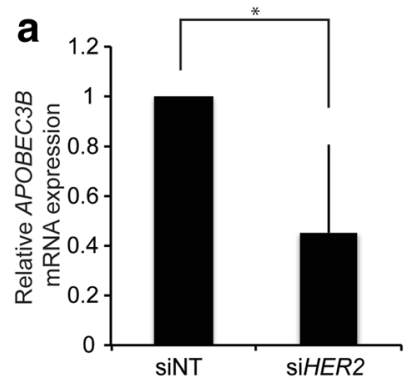

d

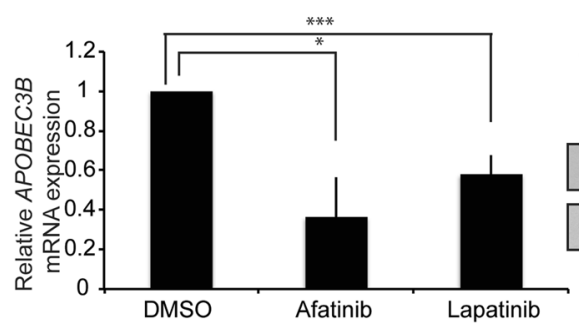

g

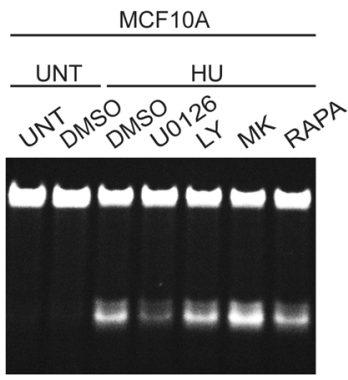

j

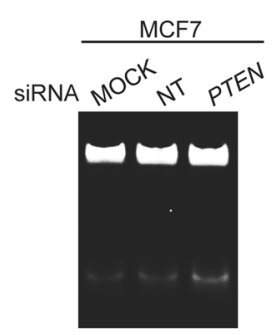

b

h

k

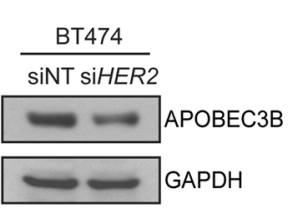

e

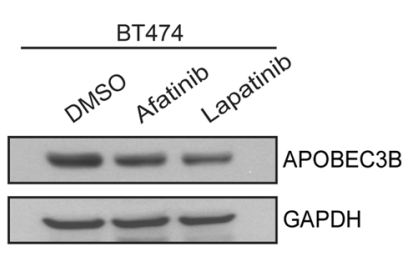

f
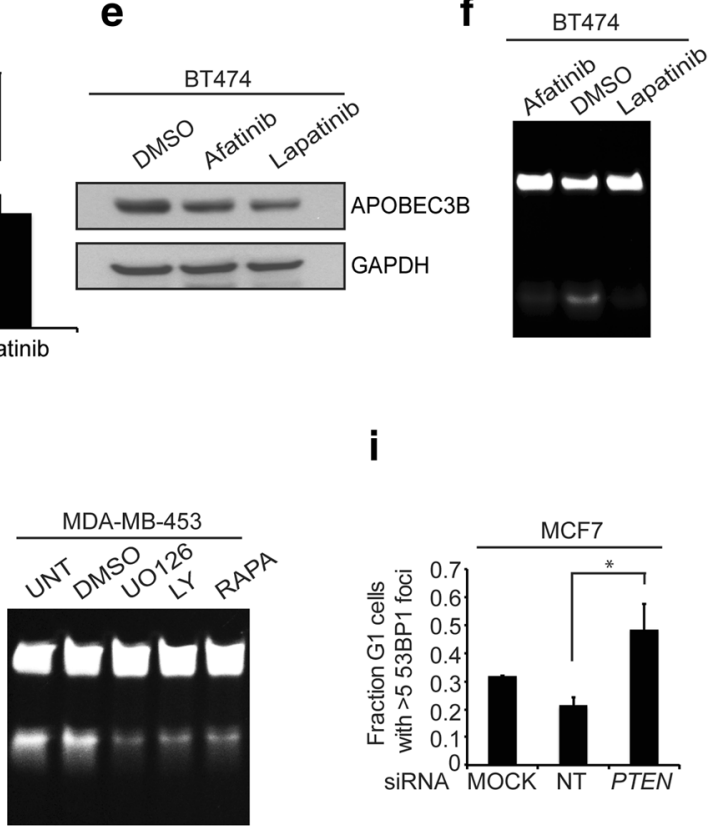

i
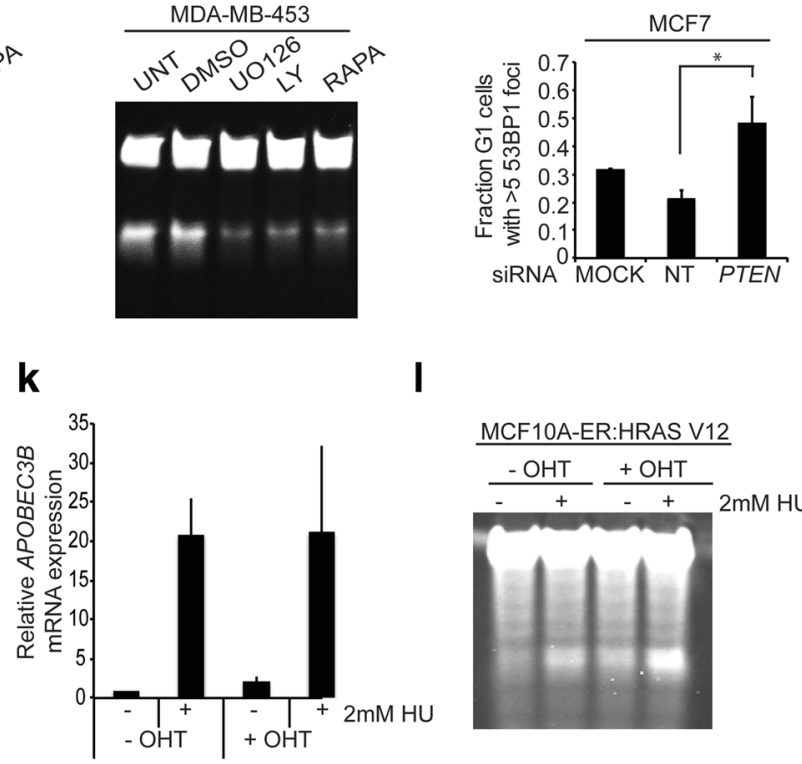

MCF10A-ER:HRAS V12

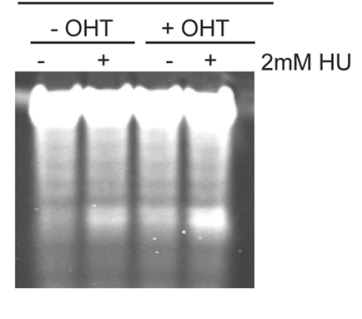

Fig. 4 (See legend on next page.) 


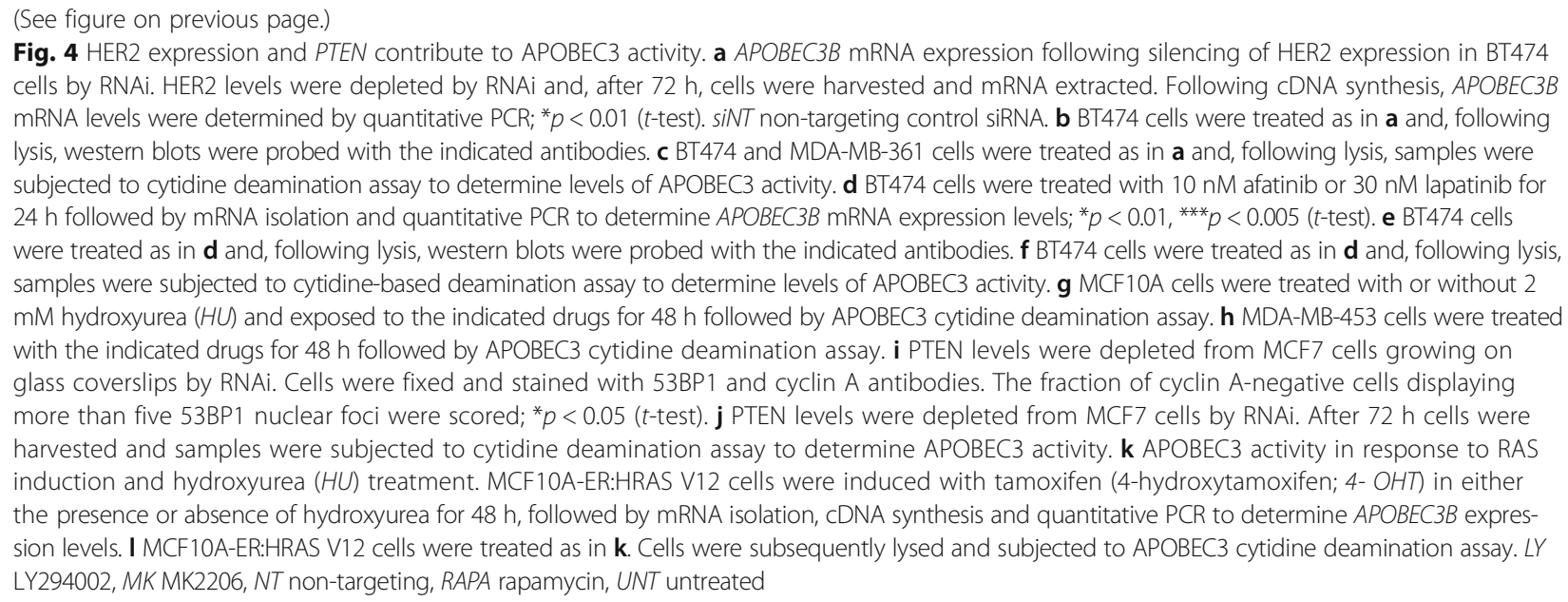

$A P O B E C 3 B$ mRNA expression (by $64 \%$ and $42 \%$, respectively), protein and deamination activity (Fig. $4 \mathrm{~d}-\mathrm{f}$; Additional file 7: Figure S7b). There was no correlation between treatment-induced cytotoxicity and APOBEC3 induction (Additional file 8: Figure S8a). Treatment with lapatinib reduced the $S$ phase population, whereas afatinib did not significantly alter the cell cycle distribution of BT474 cells (Additional file 8: Figure S8c). In addition, lapatinib treatment was able to reduce hydroxyureainduced $A P O B E C 3 B$ transcription and activity in HER2+ HCC1419 (Additional file 7: Figure S7c-f). These results suggest that signal transduction cascades downstream of HER2 could be implicated in APOBEC3 induction.

We next investigated the requirement for phosphatidylinositol 3-kinase (PI3K), mitogen-activated protein kinase (MAPK), AKT and mammalian target of rapamycin (mTOR) signalling pathways on replication stress-induced APOBEC3 activation. First MCF10A cells, with a low basal level of replication stress, were treated with hydroxyurea in the presence of inhibitors of these signalling pathways for $24 \mathrm{~h}$. Inhibition of MEK signalling (with U0126) and to a lesser extent mTOR signalling (with rapamycin) attenuated hydroxyurea-induced APOBEC3G transcription and APOBEC3 activity (Fig. 4g; Additional file 8: Figure S8d), implicating both arms of the signalling cascade in the exacerbation of DNA replication stressinduced APOBEC3 mutagenesis. Consistent with this finding, treatment of MDA-MB-453, HCC1569 and BT474 cells with U0126, rapamycin and LY294002 also led to a reduction in basal APOBEC3 activity (Fig. 4h; Additional file 8: Figure S8e, f). There was no correlation between the extent of cytotoxicity of the drugs and their ability to induce APOBEC3 activity (Additional file 8: Figure S8b; Additional file 9: Figure S9a). In BT474 cells treatment with UO126 and rapamycin appeared to reduce the G2/M phase (Additional file 8: Figure S8c). In MCF7 cells hydroxyurea appeared to induced cell cycle arrest in the $\mathrm{S}$ and G2/M phases, which could be rescued with rapamycin treatment (Additional file 9: Figure S9b). Since we identified that PTEN mutations were also associated with enrichment of the APOBEC3 mutational signature, we also investigated the effect of PTEN loss on replication stressinduced APOBEC3 activity. MCF7 cells were depleted of PTEN by siRNA and replication stress was assessed by scoring the presence of G1 nuclear bodies. Silencing of PTEN led to a significant increase in G1 bodies from 21 to $48 \%$ ( $p$ value $=0.027, t$-test; Fig. $4 \mathrm{i})$ and an increase in APOBEC3B protein and cytidine deamination activity (Fig. 4j; Additional file 9: Figure S9c, d). PTEN knockdown did not cause a significant change in cell viability or a change in cell cycle distribution that could account for the increase in APOBEC3 activity observed (Additional file 9: Figure S9e, f).

\section{Oncogene-induced replication stress and APOBEC3 activation}

Overexpression of several oncoproteins, including RAS, MYC, CCND1 and CCNE, has been demonstrated to cause increased origin firing and increased proliferation by accelerating G1/S transition leading to replication stress [3, 29-32]. To determine if oncogene-induced replication stress would also lead to APOBEC3 activation, we treated an MCF10A cell line stably expressing a 4-hydroxytamoxifen-inducible oncogenic RAS chimeric protein, ER:HRAS V12 [33], with hydroxyurea and assessed APOBEC3 activation. Treatment of MCF10A-ER:HRAS V12 cells with $2 \mathrm{mM}$ hydroxyurea increased $A P O B E C 3 B$ mRNA expression approximately 20fold, which was not further increased by the activation of RAS V12 (Fig. 4k). RAS V12 induction in the absence of hydroxyurea led to a modest increase in APOBEC3 deamination activity relative to non-induced cells (Fig. 4l). RAS V12 induction in the presence of hydroxyurea also led to a modest increase in APOBEC3 deamination activity compared with hydroxyurea treatment alone. These results 
illustrate that RAS hyperactivation alone is insufficient to significantly activate APOBEC3 in this system.

\section{ATR pathway implicated in APOBEC3 regulation}

Since DNA replication stress activates the ataxia telangiectasia mutated and Rad3-related protein (ATR)/ Checkpoint kinase 1 (Chk1) response, we investigated whether these kinases may mediate APOBEC3 activity following hydroxyurea-induced DNA replication stress. MCF10A cells were first treated with ATR and ATM kinase inhibitors and APOBEC3 activity was assessed. Inhibition of ATR and to a lesser extent ATM led to a reduction of the hydroxyurea-induced APOBEC3 activation (Fig. 5a). Furthermore, treatment of MDA-MB453 and BT474 cells with the novel specific Chk1 inhibitor CCT244747 [34] led to a reduction of basal APOBEC3 activity (Fig. 5b; Additional file 10: Figure S10a). Similarly, treatment of MCF10A cells with CCT244747 led to a reduction in hydroxyurea-induced APOBEC3B protein and activity (Fig. 5c, d). In addition, MCF10A cells were depleted of $A T R$ or CHEK1 by siRNA and subsequently APOBEC3 activity was measured under basal conditions and in response to hydroxyurea treatment. In the control siRNA transfected cells, hydroxyurea treatment caused robust APOBEC3 activation; in contrast, this response was reduced following depletion of ATR or CHEK1 (Additional file 10: Figure S10b, c). Consistent with these findings, Chk1 inhibition using an alternative Chk1 inhibitor, UCN01, also prevented the hydroxyurea-dependent increase in $A P O B E C 3 B$ transcription (Additional file 10: Figure S10d). Interestingly, $A P O B E C 3 B$ expression correlated with sensitivity to the Chk1 inhibitor CCT244747 (Additional file 10: Figure S10e). There was no correlation between drug-induced cytotoxicity or changes in cell cycle
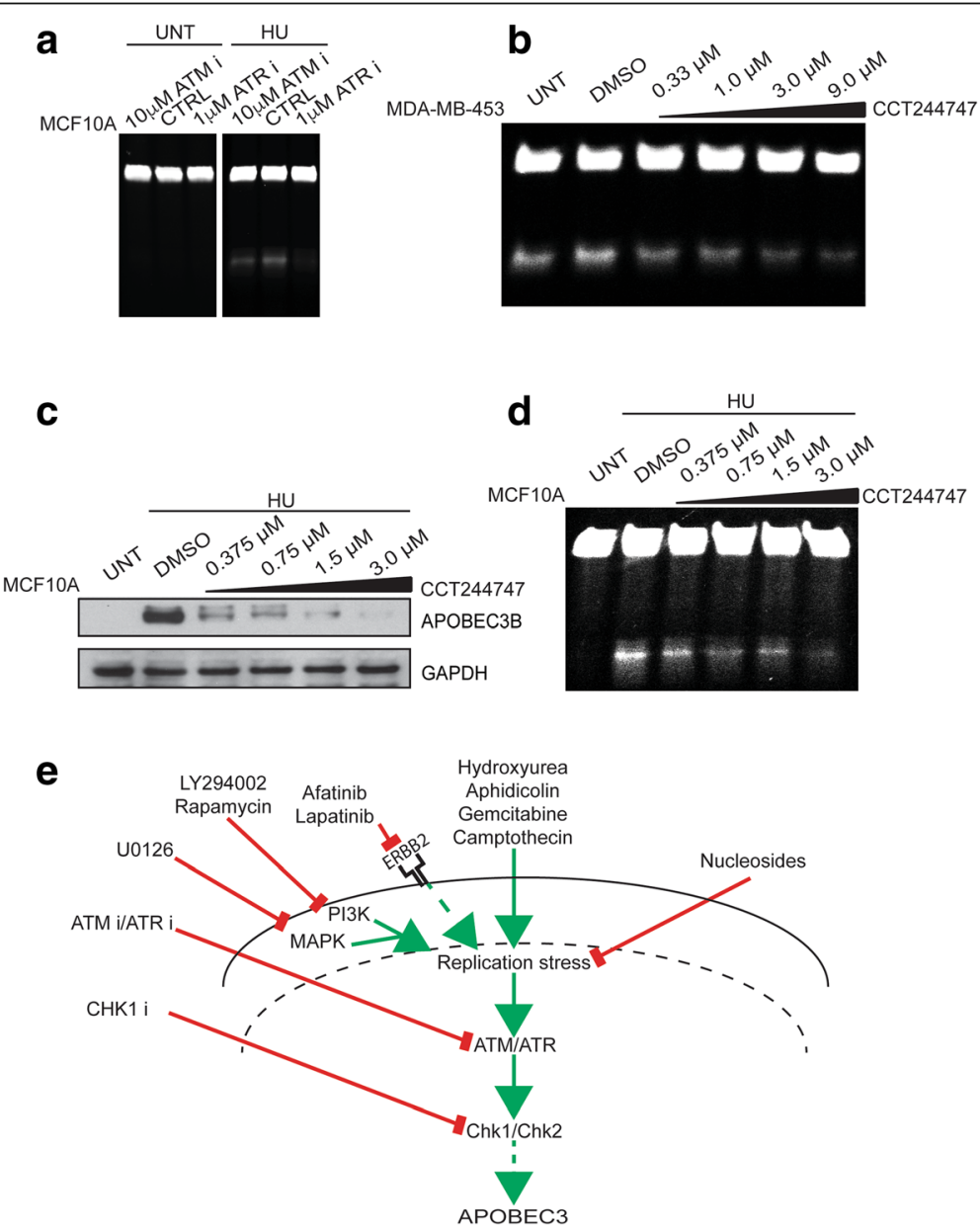

Fig. 5 DNA damage signalling and APOBEC3 activity in breast cancer cell lines. a MCF10A cells were treated with ATM or ATR inhibitors for $24 \mathrm{~h}$ after which cells were treated with hydroxyurea (HU) for a further $48 \mathrm{~h}$ prior to lysis and cytidine deamination assay for APOBEC3 activity. b MDAMB-453 cells were treated for $48 \mathrm{~h}$ with the indicated doses of Chk1 inhibitor CCT244747 followed by APOBEC3 cytidine deamination assay. c MCF10A cells were treated for $48 \mathrm{~h}$ with the indicated doses of Chk1 inhibitor CCT244747 and with $2 \mathrm{mM}$ hydroxyurea for the last $24 \mathrm{~h}$. Following lysis, western blots were probed with the indicated antibodies. $\mathbf{d}$ MCF10A cells were treated as in $\mathbf{c}$ before lysis and cytidine deamination assay to determine APOBEC3 activity. e Model illustrating mechanisms of APOBEC3 regulation by replication stress. CTRL control, UNT untreated 
distribution and APOBEC3 levels following treatment with the ATR, ATM or Chk1 inhibitors (Additional file 9: Figure S9b; Additional file 10: Figure S10f-h).

\section{Elevated replication stress-associated DNA damage in HER2-positive breast carcinomas}

Since we observed that most HER2+ cell lines exhibited high levels of $A P O B E C 3 B$ mRNA expression and replication stress-associated G1 nuclear bodies, we next investigated whether the same characteristics were observed in vivo. We performed immunohistochemical analysis on sections from formalin-fixed, paraffin-embedded material from a clinical cohort of breast carcinomas and adjacent normal breast tissue with antibodies against $\gamma \mathrm{H} 2 \mathrm{AX}$ and phosphorylated RPA (RPA-P), markers of DNA damage and ATR signalling, respectively (Additional file 11: Figure S11a). We also tested commercially available APOBEC3B antibodies for suitability in immunohistochemistry but none were sufficient to reliably and specifically detect nuclear APOBEC3B. Compared with luminal breast tumours, the HER2+ tumours exhibited significantly higher proportion of samples with more than $10 \%$ of cells staining positive for $\gamma \mathrm{H} 2 \mathrm{AX}(p=3.3 \mathrm{e}-06$, Fishers exact test; Additional file 11: Figure S11b). HER2+ tumours also exhibited more RPA-P than normal breast tissue (Additional file 11: Figure S11c). In addition, all but one case among the tumours exhibiting a high proportion of cancer cells positive for the $\gamma \mathrm{H} 2 \mathrm{AX}$ marker showed concomitant high levels of RPA-P, suggesting that replication stress contributes to the DNA damage observed in the HER2+ tumours. These results strengthen our conclusion that HER2+ tumours, which are associated with the highest mutational load of APOBEC3 mutations, exhibit high levels of replication stress-associated DNA damage.

\section{Discussion}

The APOBEC3 mutational signature has been found in a range of different cancer types and is one of the most frequently occurring mutational signatures in the cancer genome [6]. It is not clear how APOBEC3 influences tumour evolution but it is hypothesized to increase the fitness of cancer cells by providing the beneficial gain of oncogene or loss of tumour suppressor gene function [23] and is associated with the progressive acquisition of subclonal mutations later in tumour evolution in some cancer types [13]. In breast cancer, the HER2+ subtype is especially affected by TCW mutations, indicating that APOBEC3 has been active during the progression of this disease [9]. It was found that HER2-amplified [9] and HER2-overexpressing tumours [35] harbour a high SCNA load, suggesting that HER2 might underlie the formation of SCNAs and APOBEC3 activity.

In addition to the activity of APOBEC3, the localization of APOBEC3 family members is also regulated. The subcellular localization of APOBEC3 family members has been evaluated using a series of green fluorescent protein fusion constructs [36-38]. In contrast to APOBEC3B, which is predominantly nuclear, APOBEC3G is cytoplasmic under steady state conditions [36-38]. However, in lymphoma cells APOBEC3G has been shown to enter the nuclear compartment as part of a DNA damage response and promote DNA repair by activating the ataxia telangiectasia mutated (ATM) DNA damage checkpoint kinase [39]. These data suggest potential roles for multiple APOBEC3 members in response to DNA damage and replication stress.

In this study, we first investigated the correlation between basal APOBEC3 activity and HER2 status. We identified a trend towards HER2-enriched breast cancerderived cell lines having high basal APOBEC3 activity. The high levels of APOBEC3 activity in the HER2+ subtype indicates that an increased gene dosage of ERBB2 may drive APOBEC3 in breast cancer, corroborating earlier observations by Roberts and colleagues [9].

Nevertheless, apart from ERBB2 amplifications we expect other oncogenes to drive APOBEC3 activity by virtue of their impact on DNA replication stress. Oncogene activation can induce replication stress in several different ways, including interfering with the timing and number of origins firing [30,32]. Furthermore, oncoproteins such as cyclin E may also induce replication stress via inactivation of retinoblastoma protein and enhanced $S$ phase entry $[2,3]$. Overexpression of RAS causes an initial hyperproliferation accelerating G1/S phase transition and we identified that PI3K and MAPK signalling contribute to APOBEC3 activity in multiple different breast cancer cell lines. This is consistent with previous data reporting the ERK signalling pathway is involved in the regulation of APOBEC3G in human T lymphocytes [40]. We also investigated APOBEC3 activity before and after RAS hyperactivation in MCF10A-ER:HRAS cells. RAS hyperactivation modestly induced APOBEC3 activity in these cells, suggesting that it could be insufficient to robustly activate APOBEC3 in tumours on its own. Since inhibition of both PI3K and MAPK pathways was shown to reduce APOBEC3 activity, one possibility is that both signalling pathways are required to activate APOBEC3 levels. Interestingly, we identified that mutations in PTEN and NF1 were also associated with APOBEC3 mutation enrichment in the different breast cancer subtypes, further implicating PI3K and MAPK signalling in co-regulating APOBEC3 mutagenesis in breast cancer. Recent reports also show NFkB signalling plays a role in the regulation of APOBEC3B [41] and AID [42]. It is likely, therefore, that additional pathways drive APOBEC3 activity in cancer.

In addition to genetic and signalling factors, we identified that specific drugs can activate APOBEC3 activity. We found that exposure of cells to hydroxyurea, aphidicolin, 
gemcitabine and camptothecin resulted in enhanced APOBEC3 activity. These drugs were modestly associated with $\mathrm{S}$ phase enrichment. A similar observation was made in yeast after exposure to hydroxyurea [43]. These treatments also resulted in the highest levels of RPA-P, indicative of increased exposure of ssDNA. Hydroxyurea and gemcitabine can both inhibit ribonucleotide reductase, aphidicolin inhibits DNA polymerase alpha whereas camptothecin is a topoisomerase I inhibitor. As the extent of RPA-P achieved was highest with these drugs, we speculate that the differentiation between these drugs and the other tested DNA damaging agents is their ability to induce sufficient ssDNA provoking downstream DNA replication stress detection in order to surpass the threshold of ATR signalling required for APOBEC3 activation. Our findings might have clinical implications as we show that several clinically used cytotoxic drugs may induce APOBEC3 activity. Future studies should test the ability of other cytotoxic drugs to induce APOBEC3 activity and investigate whether relapse samples contain more therapy-induced APOBEC3 mutations.

Besides inducing APOBEC3 activity, we were also able to diminish APOBEC3 activation through supplementing growth media with exogenous nucleosides, which is known to rescue DNA replication stress and genomic instability [26, 27]. In addition we show that APOBEC3 activation is coordinated by a transcriptional response downstream of the key signalling cascades governed by ATR/ATM and this signalling can be rescued by exogenous nucleoside supplementation. These data implicate a role for replication-associated damage in triggering the transcriptional response to APOBEC3 activation. Interestingly, we found that two different Chk1 inhibitors potently inhibited APOBEC3 activity. Since ATR/Chk1 inhibition would lead to a failure to signal the presence of exposed ssDNA, these data are consistent with a role for this signalling cascade in mediating the DNA replication stressinvoked pathway following ssDNA exposure to APOBEC3 mRNA transcriptional activation. These findings are of potential clinical relevance as an analogue of CCT244747 will soon be tested in a phase 1 clinical trial and biomarkers of response to therapy are actively under investigation [44]. Additionally, we found a trend between $A P O B E C 3 B$ expression and sensitivity to CCT244747 $(p$ value $=0.058$, Spearman rank correlation test; Additional file 10: Figure S10e), which further strengthens the potential clinical relevance of our findings. We speculate that high levels of replication stress results in the activation of Chk1 and the subsequent induction of APOBEC3 activity. These cells exhibit high levels of DNA replication stress and are, therefore, more likely to be dependent on Chk1 signalling for repair [45], which may explain why we observe that cells with high APOBEC3 activity appear more sensitive to Chk1 inhibition. We speculate that more direct gene expression biomarkers, apart from $A P O B E C 3 B$, could potentially be of use to predict response to CCT244747. Although more research is needed, we speculate that whereas replication stress-induced $A P O B E C 3 B$ regulation could contribute to a portion of APOBEC3-mediated mutagenesis, $A P O B E C 3 G$ upregulation could enhance DNA repair, as has been previously postulated in lymphoma cells [39]. Our in vitro data are supported by our observation that replication stress-associated DNA damage was significantly associated with the HER2+ subtype, suggesting that replication stress could potentially drive APOBEC 3 activation in HER2+ breast carcinomas.

We recently showed in ER-negative breast cancer that there is a modest increase in the APOBEC3 mutation signature in later, subclonal mutations, implicating this process in the exacerbation of intratumour heterogeneity in ER-negative breast cancer [13]. Our current data suggests that this late activation could be a response to altered levels of replication stress (Fig. 5e). DNA replication stress and its induction through mitogenic signalling could be a particularly potent driver of genomic instability, inducing both chromosomal instability and APOBEC3 activation (Fig. 5e).

\section{Conclusions}

These findings illustrate that DNA replication stress appears to be a particularly potent driver of genomic instability in cancer. On the one hand, DNA replication stress has been demonstrated to induce both structural and numerical chromosomal aberrations generating chromosomal instability [27]. In addition, we now propose that replication stress is able to induce single nucleotide-level mutagenesis mediated via $A P O B E C 3 B$ induction. These dual roles for DNA replication stress in mediating genomic instability could link the high level of SCNAs and single nucleotide diversity caused by APOBEC 3 activity that are both observed in HER2+ tumours.

We have found that oncogenic signalling, cytotoxic drugs and genetic modulators of replication stress are all able to modulate APOBEC3 activity. These findings implicate the ability of therapeutics that either attenuate oncogenic signalling or exacerbate DNA replication stress to alter cancer's mutagenic landscape and evolutionary potential.

\section{Methods \\ Cell lines}

SKBR3, MCF7, HCC1419, HCC1569, HCC1954, T47D, BT474, MDA-MB-231, MDA-MB-361, MDA-MB-453, ZR75.1, MDA-MB-134, CAL51, HCC1428 cell lines were obtained from The Francis Crick Institute, Cell services. All cells were grown in RPMI 1640 media (ThermoFisher Scientific), supplemented with $10 \%$ foetal bovine srum and 1/10,000 units of penicillin-streptomycin (SigmaAldrich) and with L-glutamine (ThermoFisher Scientific). 
MCF10A cells were a kind gift from Almut Schulze. MCF10A-ER:HRAS were a kind gift from Julian Downward. MCF10A and MCF10A-ER:HRAS cells were grown in DMEMF12 supplemented with $5 \%$ horse serum, $10 \mu \mathrm{g} /$ $\mathrm{ml}$ insulin, $5 \mu \mathrm{g} / \mathrm{ml}$ hydrocortisone, $20 \mathrm{ng} / \mathrm{ml}$ epidermal growth factor, $100 \mathrm{ng} / \mathrm{ml}$ cholera toxin and 1/10000 units of penicillin-streptomycin. All cell lines used in this study were maintained at $37{ }^{\circ} \mathrm{C}$ in $5 \% \mathrm{CO}_{2}$.

\section{Treatments}

Where indicated, cells were treated with $2 \mathrm{mM}$ hydroxyurea, $2.5 \mu \mathrm{m}$ aphidicolin, $10 \mu \mathrm{m}$ etoposide, $10 \mu \mathrm{m} 5$ fluorouracil, $1 \mu \mathrm{M}$ cisplatin, $1 \mu \mathrm{M}$ gemcitabine, $0.001 \%$ methyl methanesulfonate, $100 \mathrm{nM}$ doxorubicin, $3 \mu \mathrm{M}$ camptothecin, $300 \mu \mathrm{M}$ nucleosides, $10 \mathrm{nM}$ afatinib, 30 nM lapatinib, $150 \mathrm{nM}$ rapamycin, $15 \mu \mathrm{m}$ MEK inhibitor U0126, 150 nM AKT inhibitor MK2206, $7.5 \mu \mathrm{m}$ PI3K inhibitor LY294002, $1 \mu \mathrm{m}$ ATR kinase inhibitor VE821 (AdooQ), CCT244747 (a kind gift from Prof. Ian Collins, ICR, London), $10 \mu \mathrm{m}$ ATM kinase inhibitor KU55933 (Merck, Millipore), 100 nM UCN01 Chk1/PKC $\beta$ inhibitor (Merck, Millipore), 12.5-300 $\mu \mathrm{M}$ EmbryoMax Nucleosides (Millipore).

\section{RNA interference}

All siRNA (Dharmacon, GE Healthcare) transfections were performed at $40 \mathrm{nM}$ final concentrations by reverse transfection with Lipofectamine ${ }^{\curvearrowleft}$ RNAiMax (Thermo Fisher Scientific): ATR (L-003202), CHEK1 (L-003255), ERBB2 (LU-003126), RRM1 (LU-004270), RRM2 (LU010379), RRM2B (LU-010575), PTEN (J-003023), APO$B E C 3 B$ (J-017322). Non-targeting (NT) control siRNA was used as control in all experiments.

\section{RNA extraction and reverse transcription PCR}

RNA was extracted using a Qiagen RNeasy kit and then reverse transcribed to cDNA using an AffinityScript cDNA synthesis kit (Agilent Technologies) according to the manufacturers' instructions. Quantitative PCR was performed with triplicates in 96-well plate format on the StepOnePlus Real-Time PCR system (ThermoFisher Scientific) using pre-designed TaqMan ${ }^{\odot}$ probes for $A P O B E C 3 B$ (Hs00358981_m1) and APOBEC3G (Hs00222415_m1) and ATR (Hs00992123_m1). RRM1, RRM2, RRM2B, PTEN and CHEK1 quantitative PCR was performed using QuantiTect SYBR Green PCR kits. mRNA expression levels were quantified using the comparative $\mathrm{Ct}$ method, normalized to DNA topoisomerase I (Hs00243257_m1).

Gel-based deamination assay using oligonucleotide probe We seeded 200,000 cells per well in six-well plates. Cells were allowed to adhere for $24 \mathrm{~h}$, after which they were treated with cytotoxic drugs or siRNAs for up to $72 \mathrm{~h}$. Cells were subsequently isolated and lysed in HED buffer
(25 mM HEPES, 5 mM EDTA, 10 \% glycerol, 1 mM DTT (added fresh) and protease inhibitor (added fresh)). The protein concentrations were equalized and deamination reactions were performed at $37^{\circ} \mathrm{C}$ for $3 \mathrm{~h}$ using the APOBEC3 probe 1 ( $5^{\prime}$-fluorescein-ATTATTATTATTATTCCC AATTATTTATTTATTTATTTATTT) [46] or probe 2 (5' ATTATTATTATTCGAATGGATTTATTTATTTATTTAT TTATTT-fluorescein-3') in a 10× UDG reaction buffer consisting of $1.25 \mu \mathrm{L}$ RNaseA $(0.125 \mathrm{mg} / \mathrm{mL}), 1 \mu \mathrm{L}$ probe $(0.2 \mathrm{pmol} / \mu \mathrm{L}), 16.5 \mu \mathrm{L}$ cleared lysate and uracil DNA glycosylase (UDG; New England Biolabs, 1.25 units). We added $100 \mathrm{mM} \mathrm{NaOH}$ and the sample was then incubated at $95{ }^{\circ} \mathrm{C}$ for 30 minutes to cleave the abasic sites followed by addition of formamide-based gel sample buffer. The reaction product was run on a $15 \%$ urea-TBE gel that was imaged and quantified on an ImageQuant LAS 4000. Probe 1 was used in all experiments unless stated otherwise.

\section{Cytotoxicity assay}

The cytotoxicity of CCT244747 was determined using a sulforhodamine-based growth delay assay. Cells were plated at appropriate densities into 96-well plates and allowed to attach for $36 \mathrm{~h}$. Drug treatment was from 4 to 10 days to allow drug contact for at least two doubling times followed by sulforhodamine B staining and 50 $\%$ growth inhibition (GI50) determination. GI50 values were determined using Graph Pad Prism 6 software and Spearman rank correlations were performed between GI50 values against $A P O B E C 3 B$ mRNA expression and APOBEC3 activity.

\section{Flow cytometry analysis}

Cells were washed in PBS and resuspended in PBS/0.1\% bovine serum albumin (BSA) and DNA was stained with propidium iodide. Samples were analyzed on a BD LSRFortessa X-20 cytometer (BD Biosciences) and processed in FlowJo.

\section{Cell viability assay}

Treatment-induced cytotoxicity was determined using the CellTiter-Glo luminescent cell viability kit (Promega) in accordance with the manufacturer's instructions.

\section{Protein extraction and western blotting}

Total cell lysates were generated as described previously $[47,48]$. Following SDS-PAGE, blots were probed with indicated antibodies diluted in $5 \%$ milk or BSA in Trisbuffered saline. Antibodies: HER2 (Cell Signaling \#2248), pSer473 AKT (Cell Signaling \#4060), total AKT (Cell Signaling \#2920), rabbit anti-APOBEC3B monoclonal antibody 5210-87-13 [41], HRP-conjugated anti- $\beta-G A P D H$ antibody (Abcam ab9482) and HRP-conjugated goat anti-mouse/rabbit immunoglobulins (Dako). Immobilon 
Western Chemiluminescent HRP Substrate (Millipore) was used for detection.

\section{Immunofluorescence}

Cells were treated and fixed as described previously [47]. Cells were stained with the indicated antibodies: 53BP1 (sc22760 Santa Cruz), cyclin A (in house), Ser139- $\gamma \mathrm{H} 2 \mathrm{AX}$ (Millipore 05636), RPA32/RPA2 (phosphoS4 + S8, Abcam ab87277). Anti-mouse and anti-rabbit IgG $(\mathrm{H}+\mathrm{L})$ Alexa Fluor 488, 594 and 647 secondary antibodies were used at 1:500 dilution (ThermoFisher Scientific).

\section{Archival tumour samples and immunohistochemistry}

Formalin fixed, paraffin-embedded specimens of normal breast tissue adjacent to tumour $(n=37)$ and breast carcinoma tissues $(n=120)$ from the tissue archive of the Danish Cancer Society Research Center in Copenhagen were examined. All tissue samples were collected from patients who underwent a mastectomy between 2003 and 2012. None of the patients had previously undergone surgery involving the breast and they did not receive preoperative treatment. Tumour subtype scoring of luminal $(n=66$; luminal $\mathrm{A}+\mathrm{B})$ and HER2 $(n=54)$ was performed based on estrogen receptor (ER), progesterone receptor (PGR), human epidermal receptor-2 (HER2), and an average Ki67 expression in accordance with St. Gallen International Breast Cancer Guidelines [49]. A HER2 gene copy score of two was evaluated by DNA FISH where a value $<2.2$ was considered negative and $\geq 2.2$ was considered positive. For immunohistochemical staining and analysis, the paraffin tissue sections $(4 \mu \mathrm{m})$ were deparaffinized in xylene and rehydrated in a graded series of ethanol-aqueous solutions. Antigen retrieval was carried out in $10 \mathrm{mM}$ citrate buffer ( $\mathrm{pH}$ 6.0) by heating the slides for 20 minutes in a microwave oven. Endogenous peroxidase activity was blocked by incubating the sections in $3 \%$ hydrogen peroxide in Trisbuffered saline for 10 minutes. The primary antibodies were incubated overnight. The following primary antibodies were used: mouse monoclonal anti-phosphohistone H2AX (Ser139, Millipore; diluted 1/2000) and rabbit polyclonal anti-phospho-RPA32 (Thr21, Abcam, diluted 1/250). Normal non-immune serum served as a negative control. The primary antibodies were incubated overnight, followed by detection using the Vectastain Elite kit according to the manufacturer's instructions (Vector Laboratories, Burlingame, CA, USA) and nickel sulphate enhancement without nuclear counterstaining, as described [2]. Immunostaining patterns on each slide were scored by an experienced oncopathologist based on the fraction of positive nuclear staining signals (counting a minimum of 300 epithelium or tumour cell nuclei per slide) and the threshold for scoring the categories of positivity was as follows: $<2 \%$ positive nuclei; $2-10 \%$ positive nuclei; and $>10 \%$ positive nuclei.

\section{Breast cancer subtype classification and APOBEC3 mutation pattern detection}

Data on breast cancer tumours $(n=755)$ were obtained from TCGA Research Network (http://cancergenome.nih.gov/). Tumours were previously divided into subtypes HER2, basal, luminal A, luminal B and normal based on the PAM50 method [21]. Primary data, including SNP6 copy number profiles, mutation calls and APOBEC3 enrichment values, were obtained from TCGA data version 2016_01_28. The APOBEC3 enrichment as a numeric value for the strength of APOBEC3 mutagenesis is calculated similarly to [9] as:

$$
\mathrm{E}=\frac{\text { mutations } \mathrm{TCW} X \text { contextC }}{\text { mutations } \mathrm{X} \text { X contextTCW }}
$$

The parameter 'mutationsTCW' displays the number of mutated cytosines in a TCW motif or mutated guanines in a WGA motif. 'MutationsC' represents the total number of mutated cytosines (or guanines), 'contextTCW' represents the total number of TCW (or WGA) motifs and context, the total number of cytosines (or guanines) in a specific region centred within 20 nucleotides before and 20 nucleotides after the mutated cytosines (or guanines).

Tumour samples that were significantly enriched for APOBEC3 signature mutations (Benjamini-Hochberg corrected $p$ value $<0.05)$ and fold enrichments $>2$ were classified as 'APOBEC high', and the rest as 'APOBEC low'. To test whether genes were associated with APOBEC3 enrichment, a permutation test was carried out with 100,000 permutations, randomly shuffling the labels between 'APOBEC high' and 'APOBEC low' samples. The entire cohort was first tested together to determine which genes were significantly associated, following which the test was performed again for each gene within each subtype.

\section{Additional files}

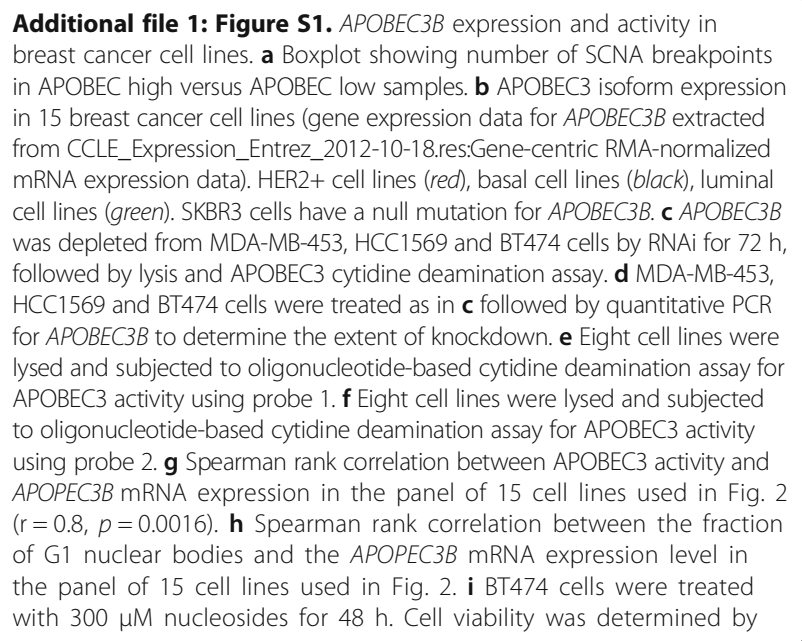

breast cancer cell lines. a Boxplot showing number of SCNA breakpoints in APOBEC high versus APOBEC low samples. b APOBEC3 isoform expression in 15 breast cancer cell lines (gene expression data for $A P O B E C 3 B$ extracted from CCLE_Expression_Entrez_2012-10-18.res:Gene-centric RMA-normalized mRNA expression data). HER2+ cell lines (red), basal cell lines (black), luminal cell lines (green). SKBR3 cells have a null mutation for APOBEC3B. c APOBEC3B was depleted from MDA-MB-453, HCC1569 and BT474 cells by RNAi for $72 \mathrm{~h}$ a for $A P O B E C 3 B$ to determine the extent of knockdown. e Eight cell lines were lysed and subjected to oligonucleotide-based cytidine deamination assay for APOBEC3 activity using probe 1. f Eight cell lines were lysed and subjected to oligonucleotide-based cytidine deamination assay for APOBEC3 activity using probe 2. g Spearman rank correlation between APOBEC3 activity and APOPEC $3 B$ mRNA expression in the panel of 15 cell lines used in Fig. of $\mathrm{G} 1$ nuclear bodies and the $A P O P E C 3 B$ mRNA expression level in with $300 \mu \mathrm{M}$ nucleosides for $48 \mathrm{~h}$. Cell viability was determined by 
CellTiter-Glo. j BT474 cells were treated with $300 \mu \mathrm{M}$ nucleosides followed by analysis of cell cycle distribution by FACS. (TIF 35303 kb)

Additional file 2: Figure S2. Induction of replication stress in cell lines using cytotoxic drugs a MCF10A cells, b MCF7 cells, c HCC1419 cells, d MDA-MB-134 cells and e SKBR3 cells were treated with the indicated drugs for $48 \mathrm{~h}$ prior to lysis and cytidine deamination assays for APOBEC3 activity. $\mathbf{f ~ M C F 7}$ and CAL51 cells were treated with siRNAs targeting APOBEC3B for $72 \mathrm{~h}$ followed by lysis and cytidine deamination assay for APOBEC3 activity. g MCF7 and CAL51 cells were treated as in $\mathbf{f}$ followed by quantitative PCR to determine levels of APOBEC3 depletion. The quantitative PCR was normalized according to siNT control. (TIF $34618 \mathrm{~kb}$ )

Additional file 3: Figure S3. Cell viability following treatment of breast cancer cell lines with cytotoxic drugs and nucleosides. a MCF10A cells, $\mathbf{b}$ MDA-MB-134 cells, c HCC1419 cells and d HCC1428 cells were incubated with the indicated drugs for $48 \mathrm{~h}$, followed by cell viability determination by CellTiter-Glo. e MCF10A cells were pre-treated with or without $300 \mu \mathrm{M}$ nucleosides for $24 \mathrm{~h}$ followed by $2 \mathrm{mM}$ hydroxyurea treatment for an additional $48 \mathrm{~h}$. Cell cycle distribution was determined by FACS analysis. (TIF $34064 \mathrm{~kb}$ )

Additional file 4: Figure S4. Cell cycle distribution following treatment of breast cancer cell lines with cytotoxic drugs. a MCF10A cells were incubated with the indicated drugs for $48 \mathrm{~h}$ prior to harvesting and determination of cell cycle distribution by FACS analysis. $\mathbf{b}$ Histogram representing the results shown in a displaying the percentage of cells in each cell cycle phase in response to the different treatments. c HCC1419 cells were incubated with the indicated drugs for $48 \mathrm{~h}$ prior to harvesting and determination of cell cycle distribution by FACS analysis. $\mathbf{d}$ MDA-MB-134 cells were incubated with the indicated drugs for $48 \mathrm{~h}$ prior to harvesting and determination of cell cycle distribution by FACS analysis. (TIF $34520 \mathrm{~kb}$ )

Additional file 5: Figure S5. Titration of cytotoxic drugs in breast cancer cell lines. a MCF10A cells were incubated with the indicated concentrations of hydroxyurea for the indicated times prior to lysis and cytidine deamination assays for APOBEC3 activity. b MCF10A cells were treated with the indicated of doses of hydroxyurea for $48 \mathrm{~h}$ prior to lysis and cytidine deamination assay for APOBEC3 activity. c MCF10A cells were treated as in $\mathbf{b}$ followed by lysis and probing western blots with the indicated antibodies. MCF10A cells were treated with the indicated concentrations of aphidicolin (d), gemcitabine (e) and cisplatin (f) for $48 \mathrm{~h}$ prior to lysis and cytidine deamination assays for APOBEC3 activity. (TIF $34567 \mathrm{~kb}$ )

Additional file 6: Figure S6. Replication stress correlates with $A P O B E C 3$ activation. a MCF7 cells were treated with the indicated drugs for $48 \mathrm{~h}$ followed by fixation and immunofluorescence for Ser139 $\mathrm{YH} 2 \mathrm{AX}$ and S4/8 RPA phosphorylation. Red asterisks indicate treatments inducing APOBEC3 activity levels and S4/8 RPA phosphorylation. Spearman rank correlations between $\mathbf{b}$ the extent of RPA phosphorylation and $A P O B E C 3 B$ mRNA expression, $\mathbf{c}$ the extent of RPA phosphorylation and APOBEC3G mRNA expression, $\mathbf{d}$ the extent of RPA phosphorylation and APOBEC3 activity (arbitrary units) from MCF10A cells treated in Fig. 3. e Quantitative PCR validation of knockdown of RRM subunits to accompany Fig. $3 f .72 \mathrm{~h}$ after siRNA transfection, MCF10A cells were lysed and mRNA isolated followed by CDNA synthesis. Quantitative PCR was performed to determine expression levels of RRM1, RRM2 and RRM2B. (TIF $38074 \mathrm{~kb}$ )

Additional file 7: Figure S7. Role of receptor tyrosine kinase signalling in APOBEC3 activation. a BT474 and MDA-MB-361 cells were treated with RNAi targeting ERBB2. After $72 \mathrm{~h}$, cells were harvested, lysed and western blots were probed with the indicated antibodies to determine the extent of ERBB2 silencing. b BT474 cells were treated with $10 \mathrm{nM}$ afatinib or 30 $\mathrm{nM}$ lapatinib for $24 \mathrm{~h}$ followed by lysis. Western blots were probed with the indicated antibodies. c HCC1419 cells were treated with $2 \mathrm{mM}$ hydroxyurea in the presence or absence of $30 \mathrm{nM}$ lapatinib. Following mRNA isolation and CDNA synthesis, $A P O B E C 3 B$ mRNA expression levels were determined by quantitative PCR. $\mathbf{d}$ HCC1419 cells were treated as in c and, following lysis, oligonucleotide-based cytidine deamination assays were performed for APOBEC3 activity using probe 1. e HCC1419 cells were treated as in c and, following lysis, APOBEC3 cytidine deamination assays were performed using probe 2 . $\mathbf{f} \mathrm{HCC} 1419$ cells were treated as in c. Cells were lysed and western blots were probed with the indicated antibodies. (TIF $34667 \mathrm{~kb}$ )

Additional file 8: Figure S8. Role of MAPK and PI3K pathways in APOBEC 3 activation. a BT474 cells were treated with $10 \mathrm{nM}$ afatinib or 30 $\mathrm{nM}$ lapatinib for $24 \mathrm{~h}$ followed by cell viability determination by CellTiter-Glo. b BT474 cells were treated with the indicated drugs for $48 \mathrm{~h}$ followed by cell viability determination by CellTiter-Glo. c BT474 cells were treated as in $\mathbf{a}$ and $\mathbf{b}$ followed by analysis of cell cycle distribution by FACS. $\mathbf{d}$ MCF10A cells were treated with the indicated drugs as in Fig. $4 \mathrm{~g}$ followed by RNA isolation, CDNA synthesis and quantitative PCR to determine APOBEC3G levels. e BT474 cells and $\mathbf{f} \mathrm{HCC} 1569$ cells were treated with the indicated drugs for $48 \mathrm{~h}$. Cells were lysed and oligonucleotide-based cytidine deamination assays were performed for APOBEC3 activity. (TIF $37363 \mathrm{~kb}$ )

Additional file 9: Figure S9. Role of MAPK and PI3K pathways in hydroxyurea-induced APOBEC3 activation. a MCF10A cells were treated with the indicated drugs for $48 \mathrm{~h}$ followed by cell viability determination by CellTiter-Glo. b MCF7 cells were treated with the indicated drugs for $48 \mathrm{~h}$ followed by analysis of cell cycle distribution by FACS analysis. c MCF7 cells were transfected with PTEN siRNA. After $72 \mathrm{~h}$ the extent of knockdown was determined by quantitative PCR. $\mathbf{d}$ PTEN levels were depleted from MCF7 cells by RNAi. After $72 \mathrm{~h}$ cells were harvested and western blots were probed with the indicated antibodies. e MCF7 cells were transfected with PTEN siRNA or ubiquitin (UBB) control, followed by cell viability determination by CellTiter-Glo. f MCF7 cells were treated as in $\mathbf{c}$ followed by cell cycle distribution analysis by FACS. (TIF 35459 kb)

Additional file 10: Figure S10. Role for DNA damage signalling in APOBEC 3 activation. a BT474 cells were treated with the indicated doses of Chk1 inhibitor CCT244747 followed by APOBEC3 cytidine deamination assay. b ATR and CHEK1 were depleted from MCF10A cells by RNAi. After 24-h transfection, cells were treated with hydroxyurea for a further $48 \mathrm{~h}$ prior to lysis and cytidine deamination assay for APOBEC3 actvity. $\mathbf{c}$ Validation of the extent of silencing of ATR and CHEK1 in MCF10A cells. Cells were treated with the indicated siRNAs for $72 \mathrm{~h}$ followed by mRNA isolation, CDNA synthesis and quantitative PCR for ATR and CHEK1 mRNA expression levels. $\mathbf{d}$ MCF10A cells were treated with hydroxyurea in the presence or absence of Chk1 inhibitor UCN-01 for $48 \mathrm{~h}$ prior to mRNA isolation, CDNA synthesis and quantitative PCR for APOBEC BB levels. e Eight breast cancer cell lines were treated with ten doses of CCT244747 for two population doublings followed by sulforhodamine B staining and GI50 determination. $\mathrm{f} \mathrm{MCF10A} \mathrm{cells} \mathrm{were}$ treated with the indicated drugs for $48 \mathrm{~h}$ followed by cell viability determination using CellTiter-Glo. g BT474 cells were treated with 9 $\mu \mathrm{M}$ CCT244747 followed by analysis of cell cycle distribution by FACS. h MCF10A cells were treated with the indicated drugs for $48 \mathrm{~h}$ followed by analysis of cell cycle distribution by FACS. (TIF $34524 \mathrm{~kb}$ )

Additional file 11: Figure S11. The HER2+ breast cancer subtype exhibits high levels of $\mathrm{YH} 2 \mathrm{AX}$ and RPA-P staining. a Examples of immunohistochemical staining of normal breast and HER2+ tumour samples with Ser139 $\mathrm{YH} 2 \mathrm{AX}$ and RPA-P. $\mathbf{b}$ The percentages of cases scoring $<2 \%, 2-10 \%$ and $>10 \%$ or more positive for Ser139 $\mathrm{YH} 2 \mathrm{AX}$ in each subtype. $P=3.314 \mathrm{e}-06$, Fishers exact test, comparing luminal versus HER2+ subtype exhibiting more than $10 \%$ of cells staining positive for Ser139 $\mathrm{YH} 2 \mathrm{AX}$. c The percentages of cases scoring $<2 \%$, 2-10\% and $>10 \%$ or more positive for RPA-P in each subtype. (TIF $34565 \mathrm{~kb}$ )

\section{Abbreviations}

APOBEC3: Apolipoprotein B mRNA editing enzyme, catalytic polypeptide-like 3; ATM: Ataxia telangiectasia mutated; BSA: Bovine serum albumin; CCLE: Cancer Cell Line Encyclopedia; DSB: double strand break; ER: Estrogen receptor; FDR: False discovery rate; GI50: 50 \% growth inhibition; HER2: Human epidermal receptor-2; MAPK: Mitogen-activated protein kinase; mTOR: Mammalian target of rapamycin; PI3K: Phosphatidylinositol 3-kinase; RNAi: RNA interference; RPA: Replication protein A; RPA-P: Phosphorylated RPA; SCNA: Somatic copy number aberration; siRNA: Small interfering RNA; ssDNA: Single-stranded DNA; TCGA: The Cancer Genome Atlas

\section{Acknowledgements}

We thank Dr Tim Fenton, Dr Eva Grönroos and our colleagues for critical discussions of this work, and Vera Timmermans Wielenga, Department of Pathology, Center of Diagnostic Investigations, Copenhagen University Hospital, 
Copenhagen, for help with sample collection for the immunohistochemistry cohort. The results published here are in part based upon data generated by The Cancer Genome Atlas pilot project established by the NCl and the National Human Genome Research Institute. Information about TCGA and the investigators and institutions who constitute TCGA research network can be found at http://cancergenome.nih.gov/ (dbGaP accession number phs000178.v9.p8). We thank all funding boards for their continued support.

\section{Funding}

C. Swanton is Royal Society Napier Research Professor. This work was supported by the Francis Crick Institute which receives its core funding from Cancer Research UK (FC001169), the UK Medical Research Council (FC001169), and the Wellcome Trust (FC001169); by the UK Medical Research Council (grant reference MR/FC001169 /1); C. Swanton is funded by Cancer Research UK (TRACERx), the CRUK Lung Cancer Centre of Excellence, Stand Up 2 Cancer (SU2C), the Rosetrees Trust, NovoNordisk Foundation (ID 16584), the Prostate Cancer Foundation, the Breast Cancer Research Foundation, the European Research Council (THESEUS) and Support was provided to C. Swanton by the National Institute for Health Research, the University College London Hospitals Biomedical Research Centre, and the Cancer Research UK University College London Experimental Cancer Medicine Centre; NK is funded by the Breast Cancer Research Foundation and Stand Up 2 Cancer. MAC and MD are funded by the Breast Cancer Research Foundation. SV is funded by Stand Up 2 Cancer. GG is funded by the Prostate Cancer Foundation. NM is funded by Cancer Research UK (TRACERX). LPZ is funded by a Cancer Research UK fellowship. Cancer research in the Harris laboratory is supported by grants from the Department of Defense Breast Cancer Research Program (BC121347), Jimmy V Foundation for Cancer Research, Minnesota Ovarian Cancer Alliance and Randy Shaver Cancer Research and Community Fund. RSH is an Investigator of the Howard Hughes Medical Institute. The Bartek laboratory is funded by the Danish Cancer Society, the Novo Nordisk Foundation (grant 16584), the Danish Council for Independent Research (DFF-1331-00262B), The Danish National Research Foundation (DNRF125, project CARD) and the Swedish Research Council and CancerFonden (grant 150733). MIW is funded by a CRUK programme grant to the Cancer Therapeutics Unit (C309/A11566). RR is funded by a Wellcome Trust studentship.

\section{Authors' contributions}

Conception and design: NK, SV, CS. Development of methodology: NK, SV, Jirina B, EKL, IG, OWR, Jiri B, RSH. Acquisition of data: NK, SV, MAC, LPZ, RR, MIW. Analysis and interpretation of data (e.g., statistical analysis, biostatistics, computational analysis): GG, NM, MD, MK. Writing, review and/or revision of the manuscript: NK, SV, Jiri B, RSH, CS. Study supervision: CS. All authors read and approved the final manuscript.

\section{Competing interests}

RSH is a co-founder of ApoGen Biotechnologies Inc. CS is a scientific advisory board member of ApoGen Biotechnologies Inc.

\section{Ethics approval and consent to participate}

This study was conducted in compliance with the Helsinki II Declaration and written informed consent was obtained from all participants. The project was approved by the Copenhagen and Frederiksberg regional division of the Danish National Committee on Biomedical Research Ethics (KF 01-069/03).

\section{Author details}

${ }^{1}$ UCL Cancer Institute, CRUK Lung Cancer Centre of Excellence, Paul O'Gorman Building, Huntley St., London, UK. ${ }^{2}$ Translational Cancer Therapeutics Laboratory, The Francis Crick Institute, London, UK. ${ }^{3}$ Danish Cancer Society Research Center, Copenhagen, Denmark. ${ }^{4}$ Department of Medical Biochemistry and Biophysics, Division of Translational Medicine and Chemical Biology, Karolinska Institute, Stockholm, Sweden. ${ }^{5}$ CRUK Cancer Therapeutics Unit, The Institute of Cancer Research, London, UK. ${ }^{6}$ Howard Hughes Medical Institute, Masonic Cancer Center, Institute for Molecular Virology, Center for Genome Engineering, Department of Biochemistry, Molecular Biology, and Biophysics, University of Minnesota, Minneapolis, Minnesota, USA. 'Department of Mathematics and Technology, University of Applied Sciences Koblenz, RheinAhrCampus Remagen, Joseph-Rovan-Allee 2, D-53424 Remagen, Germany.
Received: 2 July 2016 Accepted: 9 August 2016 Published online: 15 September 2016

\section{References}

1. Hanahan D, Weinberg RA. Hallmarks of cancer: the next generation. Cell. 2011;144:646-74.

2. Bartkova J, Horejsi Z, Koed K, Kramer A, Tort F, Zieger K, Guldberg P, Sehested M, Nesland JM, Lukas $C$, et al. DNA damage response as a candidate anti-cancer barrier in early human tumorigenesis. Nature. 2005;434:864-70.

3. Bartkova J, Rezaei N, Liontos M, Karakaidos P, Kletsas D, Issaeva N, Vassiliou LV, Kolettas E, Niforou K, Zoumpourlis VC, et al. Oncogene-induced senescence is part of the tumorigenesis barrier imposed by DNA damage checkpoints. Nature. 2006:444:633-7.

4. Burrell RA, McGranahan N, Bartek J, Swanton C. The causes and consequences of genetic heterogeneity in cancer evolution. Nature. 2013;501:338-45.

5. Gerlinger M, McGranahan N, Dewhurst SM, Burrell RA, Tomlinson I, Swanton C. Cancer: evolution within a lifetime. Annu Rev Genet. 2014;48:215-36.

6. Alexandrov LB, Nik-Zainal S, Wedge DC, Aparicio SA, Behjati S, Biankin AV, Bignell GR, Bolli N, Borg A, Borresen-Dale AL, et al. Signatures of mutational processes in human cancer. Nature. 2013;500:415-21.

7. Burns MB, Temiz NA, Harris RS. Evidence for APOBEC3B mutagenesis in multiple human cancers. Nat Genet. 2013;45:977-83.

8. Nik-Zainal S, Alexandrov LB, Wedge DC, Van Loo P, Greenman CD, Raine K, Jones D, Hinton J, Marshall J, Stebbings LA, et al. Mutational processes molding the genomes of 21 breast cancers. Cell. 2012;149:979-93.

9. Roberts SA, Lawrence MS, Klimczak L, Grimm SA, Fargo D, Stojanov P, Kiezun A, Kryukov GV, Carter SL, Saksena G, et al. An APOBEC cytidine deaminase mutagenesis pattern is widespread in human cancers. Nat Genet. 2013;45:970-6.

10. Burns MB, Lackey L, Carpenter MA, Rathore A, Land AM, Leonard B, Refsland EW, Kotandeniya D, Tretyakova N, Nikas JB, et al. APOBEC3B is an enzymatic source of mutation in breast cancer. Nature. 2013;494:366-70.

11. Taylor BJ, Nik-Zainal S, Wu YL, Stebbings LA, Raine K, Campbell PJ, Rada C, Stratton MR, Neuberger MS. DNA deaminases induce break-associated mutation showers with implication of $\mathrm{APOBEC} 3 \mathrm{~B}$ and $3 \mathrm{~A}$ in breast cancer kataegis. Elife. 2013;2, e00534.

12. de Bruin EC, McGranahan N, Mitter R, Salm M, Wedge DC, Yates L, JamalHanjani M, Shafi S, Murugaesu N, Rowan AJ, et al. Spatial and temporal diversity in genomic instability processes defines lung cancer evolution. Science. 2014;346:251-6.

13. McGranahan N, Favero F, de Bruin EC, Birkbak NJ, Szallasi Z, Swanton C. Clonal status of actionable driver events and the timing of mutational processes in cancer evolution. Sci Transl Med. 2015;7:283ra254.

14. Rosenthal R, McGranahan N, Herrero J, Taylor BS, Swanton C. DeconstructSigs: delineating mutational processes in single tumors distinguishes DNA repair deficiencies and patterns of carcinoma evolution. Genome Biol. 2016;17:31.

15. Sakofsky CJ, Roberts SA, Malc E, Mieczkowski PA, Resnick MA, Gordenin DA, Malkova A. Break-induced replication is a source of mutation clusters underlying kataegis. Cell Rep. 2014;7:1640-8.

16. Roberts SA, Sterling J, Thompson C, Harris S, Mav D, Shah R, Klimczak LJ, Kryukov GV, Malc E, Mieczkowski PA, et al. Clustered mutations in yeast and in human cancers can arise from damaged long single-strand DNA regions. Mol Cell. 2012:46:424-35.

17. Zeman MK, Cimprich KA. Causes and consequences of replication stress. Nat Cell Biol. 2014;16:2-9.

18. Jeggo PA, Geuting V, Lobrich $M$. The role of homologous recombination in radiation-induced double-strand break repair. Radiother Oncol. 2011;101:7-12.

19. Gan W, Guan Z, Liu J, Gui T, Shen K, Manley JL, Li X. R-loop-mediated genomic instability is caused by impairment of replication fork progression. Genes Dev. 2011;25:2041-56.

20. Maciejowski J, Li Y, Bosco N, Campbell PJ, de Lange T. Chromothripsis and kataegis induced by telomere crisis. Cell. 2015;163:1641-54.

21. Ciriello G, Gatza ML, Beck AH, Wilkerson MD, Rhie SK, Pastore A, Zhang H, McLellan M, Yau C, Kandoth C, et al. Comprehensive molecular portraits of invasive lobular breast cancer. Cell. 2015;163:506-19.

22. Parker JS, Mullins M, Cheang MC, Leung S, Voduc D, Vickery T, Davies $S$, Fauron $\mathrm{C}, \mathrm{He} \mathrm{X}, \mathrm{Hu} \mathrm{Z}$, et al. Supervised risk predictor of breast cancer based on intrinsic subtypes. J Clin Oncol. 2009;27:1160-7. 
23. Henderson S, Chakravarthy A, Su X, Boshoff C, Fenton TR. APOBEC-mediated cytosine deamination links PIK3CA helical domain mutations to human papillomavirus-driven tumor development. Cell Rep. 2014;7:1833-41.

24. Moasser MM. The oncogene HER2: its signaling and transforming functions and its role in human cancer pathogenesis. Oncogene. 2007;26:6469-87.

25. Lukas C, Savic V, Bekker-Jensen S, Doil C, Neumann B, Pedersen RS, Grofte M, Chan KL, Hickson ID, Bartek J, Lukas J. 53BP1 nuclear bodies form around DNA lesions generated by mitotic transmission of chromosomes under replication stress. Nat Cell Biol. 2011;13:243-53.

26. Bester AC, Roniger M, Oren YS, Im MM, Sarni D, Chaoat M, Bensimon A, Zamir G, Shewach DS, Kerem B. Nucleotide deficiency promotes genomic instability in early stages of cancer development. Cell. 2011;145:435-46.

27. Burrell RA, McClelland SE, Endesfelder D, Groth P, Weller MC, Shaikh N, Domingo E, Kanu N, Dewhurst SM, Gronroos E, et al. Replication stress links structural and numerical cancer chromosomal instability. Nature. 2013;494:492-6.

28. Sampath D, Rao VA, Plunkett W. Mechanisms of apoptosis induction by nucleoside analogs. Oncogene. 2003;22:9063-74

29. Aggarwal P, Lessie MD, Lin DI, Pontano L, Gladden AB, Nuskey B, Goradia A, Wasik MA, Klein-Szanto AJ, Rustgi AK, et al. Nuclear accumulation of cyclin D1 during S phase inhibits Cul4-dependent Cdt1 proteolysis and triggers p53-dependent DNA rereplication. Genes Dev. 2007;21:2908-22.

30. Gaillard H, Garcia-Muse T, Aguilera A. Replication stress and cancer. Nat Rev Cancer. 2015;15:276-89.

31. Hills SA, Diffley JF. DNA replication and oncogene-induced replicative stress. Curr Biol. 2014;24:R435-44.

32. Maya-Mendoza A, Ostrakova J, Kosar M, Hall A, Duskova P, Mistrik M, MerchutMaya JM, Hodny Z, Bartkova J, Christensen C, Bartek J. Myc and Ras oncogenes engage different energy metabolism programs and evoke distinct patterns of oxidative and DNA replication stress. Mol Oncol. 2015;9:601-16.

33. Molina-Arcas M, Hancock DC, Sheridan C, Kumar MS, Downward J. Coordinate direct input of both KRAS and IGF1 receptor to activation of PI3 kinase in KRAS-mutant lung cancer. Cancer Discov. 2013;3:548-63.

34. Walton Ml, Eve PD, Hayes A, Valenti MR, De Haven Brandon AK, Box G, Hallsworth A, Smith EL, Boxall KJ, Lainchbury M, et al. CCT244747 is a novel potent and selective CHK1 inhibitor with oral efficacy alone and in combination with genotoxic anticancer drugs. Clin Cancer Res. 2012;18:5650-61.

35. Rowald K, Mantovan M, Passos J, Buccitelli C, Mardin BR, Korbel JO, Jechlinger M, Sotillo R. Negative selection and chromosome instability induced by Mad2 overexpression delay breast cancer but facilitate oncogene-independent outgrowth. Cell Rep. 2016;15:2679-91.

36. Hultquist JF, Lengyel JA, Refsland EW, LaRue RS, Lackey L, Brown WL, Harris RS. Human and rhesus APOBEC3D, APOBEC3F, APOBEC3G, and APOBEC3H demonstrate a conserved capacity to restrict Vif-deficient HIV-1. J Virol. 2011;85:11220-34.

37. Lackey L, Law EK, Brown WL, Harris RS. Subcellular localization of the APOBEC3 proteins during mitosis and implications for genomic DNA deamination. Cell Cycle. 2013;12:762-72.

38. Land AM, Law EK, Carpenter MA, Lackey L, Brown WL, Harris RS. Endogenous APOBEC3A DNA cytosine deaminase is cytoplasmic and nongenotoxic. J Biol Chem. 2013;288:17253-60.

39. Nowarski R, Wilner Ol, Cheshin O, Shahar OD, Kenig E, Baraz L, Britan-Rosich E, Nagler A, Harris RS, Goldberg M, et al. APOBEC3G enhances lymphoma cell radioresistance by promoting cytidine deaminase-dependent DNA repair. Blood. 2012;120:366-75.

40. Rose KM, Marin M, Kozak SL, Kabat D. Transcriptional regulation of APOBEC3G, a cytidine deaminase that hypermutates human immunodeficiency virus. J Biol Chem. 2004;279:41744-9.

41. Leonard B, McCann JL, Starrett GJ, Kosyakovsky L, Luengas EM, Molan AM, Burns MB, McDougle RM, Parker PJ, Brown WL, Harris RS. The PKC/NF-kappaB signaling pathway induces APOBEC3B expression in multiple human cancers. Cancer Res. 2015;75:4538-47.

42. Matsumoto Y, Marusawa H, Kinoshita K, Endo Y, Kou T, Morisawa T, Azuma T, Okazaki IM, Honjo T, Chiba T. Helicobacter pylori infection triggers aberrant expression of activation-induced cytidine deaminase in gastric epithelium. Nat Med. 2007;13:470-6.

43. Hoopes JI, Cortez LM, Mertz TM, Malc EP, Mieczkowski PA, Roberts SA. $A P O B E C 3 A$ and $A P O B E C 3 B$ preferentially deaminate the lagging strand template during DNA replication. Cell Rep. 2016;14:1273-82.
44. Walton MI, Eve PD, Hayes A, Henley AT, Valenti MR, De Haven Brandon AK, Box G, Boxall KJ, Tall M, Swales K, et al. The clinical development candidate CCT245737 is an orally active CHK1 inhibitor with preclinical activity in RAS mutant NSCLC and Emicro-MYC driven B-cell lymphoma. Oncotarget. 2016;7:2329-42

45. Syljuasen RG, Sorensen CS, Hansen LT, Fugger K, Lundin C, Johansson F, Helleday T, Sehested M, Lukas J, Bartek J. Inhibition of human Chk1 causes increased initiation of DNA replication, phosphorylation of ATR targets, and DNA breakage. Mol Cell Biol. 2005;25:3553-62.

46. Opi S, Takeuchi H, Kao S, Khan MA, Miyagi E, Goila-Gaur R, Iwatani Y, Levin JG, Strebel K. Monomeric APOBEC3G is catalytically active and has antiviral activity. J Virol. 2006;80:4673-82.

47. Kanu N, Zhang T, Burrell RA, Chakraborty A, Cronshaw J, Costa CD, Gronroos E, Pemberton HN, Anderton E, Gonzalez L, et al. RAD18, WRNIP1 and ATMIN promote ATM signalling in response to replication stress. Oncogene. 2015; 35:4009-19.

48. Kanu N, Gronroos E, Martinez P, Burrell RA, Yi Goh X, Bartkova J, MayaMendoza A, Mistrik M, Rowan AJ, Patel H, et al. SETD2 loss-of-function promotes renal cancer branched evolution through replication stress and impaired DNA repair. Oncogene. 2015;34:5699-708.

49. Coates AS, Winer EP, Goldhirsch A, Gelber RD, Gnant M, Piccart-Gebhart M, Thurlimann B, Senn HJ, Panel M. Tailoring therapies-improving the management of early breast cancer: St Gallen International Expert Consensus on the Primary Therapy of Early Breast Cancer 2015. Ann Oncol. 2015;26:1533-46.

\section{Submit your next manuscript to BioMed Central and we will help you at every step:}

- We accept pre-submission inquiries

- Our selector tool helps you to find the most relevant journal

- We provide round the clock customer support

- Convenient online submission

- Thorough peer review

- Inclusion in PubMed and all major indexing services

- Maximum visibility for your research

Submit your manuscript at www.biomedcentral.com/submit
) Biomed Central 